\title{
Vortex identification from local properties of the vorticity field
}

J. H. Elsas and L. Moriconi

Citation: Physics of Fluids 29, 015101 (2017); doi: 10.1063/1.4973243

View online: http://dx.doi.org/10.1063/1.4973243

View Table of Contents: http://aip.scitation.org/toc/phf/29/1

Published by the American Institute of Physics

\section{Articles you may be interested in}

A few thoughts on proper orthogonal decomposition in turbulence

Physics of Fluids 29, 020709020709 (2017); 10.1063/1.4974330

Modulating flow and aerodynamic characteristics of a square cylinder in crossflow using a rear jet injection Physics of Fluids 29, 015103015103 (2017); 10.1063/1.4972982

Effects of grid geometry on non-equilibrium dissipation in grid turbulence

Physics of Fluids 29, 015102015102 (2017); 10.1063/1.4973416

Effect of trailing edge shape on the separated flow characteristics around an airfoil at low Reynolds number:

A numerical study

Physics of Fluids 29, 014101014101 (2017); 10.1063/1.4973811

Referee Acknowledgment for 2016

Physics of Fluids 29, 010201010201 (2017); 10.1063/1.4974753

Buoyancy effects in an unstably stratified turbulent boundary layer flow

Physics of Fluids 29, 015104015104 (2017); 10.1063/1.4973667

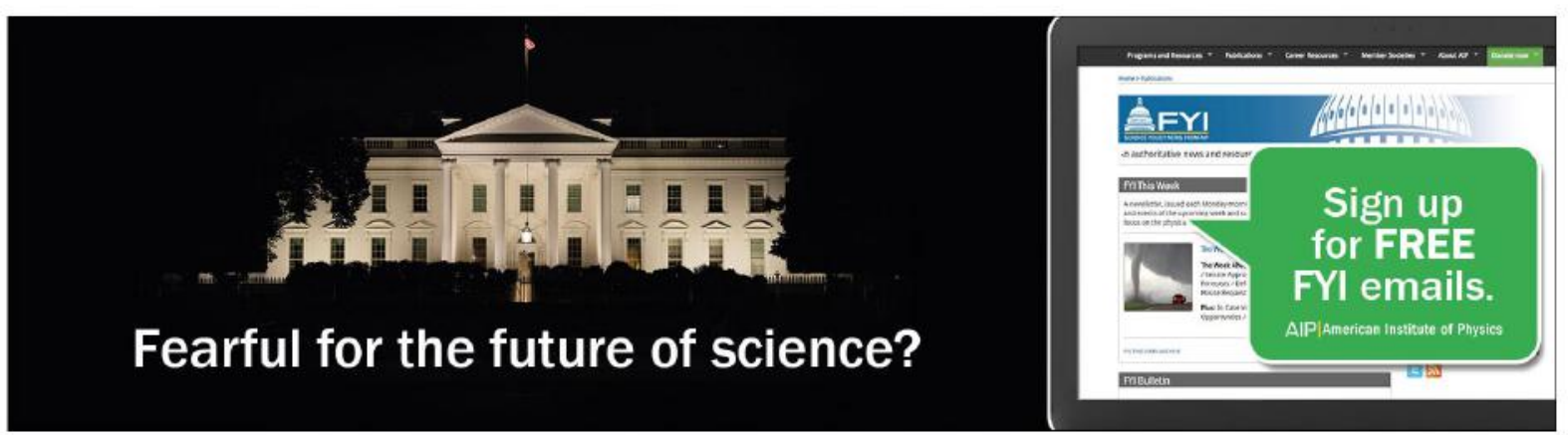




\title{
Vortex identification from local properties of the vorticity field
}

\author{
J. H. Elsas ${ }^{1,2}$ and L. Moriconi ${ }^{2}$ \\ ${ }^{1}$ Department of Mechanical Engineering, The Johns Hopkins University, Baltimore, Maryland 21218, USA \\ ${ }^{2}$ Instituto de Física, Universidade Federal do Rio de Janeiro, C.P. 68528, 21945-970 Rio de Janeiro, RJ, Brazil
}

(Received 14 July 2016; accepted 13 December 2016; published online 3 January 2017)

\begin{abstract}
A number of systematic procedures for the identification of vortices/coherent structures have been developed as a way to address their possible kinematical and dynamical roles in structural formulations of turbulence. It has been broadly acknowledged, however, that vortex detection algorithms, usually based on linear-algebraic properties of the velocity gradient tensor, can be plagued with severe shortcomings and may become, in practical terms, dependent on the choice of subjective threshold parameters in their implementations. In two-dimensions, a large class of standard vortex identification prescriptions turn out to be equivalent to the "swirling strength criterion" ( $\lambda_{c i}$-criterion), which is critically revisited in this work. We classify the instances where the accuracy of the $\lambda_{c i}$-criterion is affected by nonlinear superposition effects and propose an alternative vortex detection scheme based on the local curvature properties of the vorticity graph $(x, y, \omega)$ - the "vorticity curvature criterion" ( $\lambda_{\omega}$-criterion) — which improves over the results obtained with the $\lambda_{c i}$-criterion in controlled Monte Carlo tests. A particularly problematic issue, given its importance in wall-bounded flows, is the eventual inadequacy of the $\lambda_{c i}$-criterion for many-vortex configurations in the presence of strong background shear. We show that the $\lambda_{\omega}$-criterion is able to cope with these cases as well, if a subtraction of the mean velocity field background is performed, in the spirit of the Reynolds decomposition procedure. A realistic comparative study for vortex identification is then carried out for a direct numerical simulation of a turbulent channel flow, including a three-dimensional extension of the $\lambda_{\omega}$-criterion. In contrast to the $\lambda_{c i}$-criterion, the $\lambda_{\omega}$-criterion indicates in a consistent way the existence of small scale isotropic turbulent fluctuations in the logarithmic layer, in consonance with long-standing assumptions commonly taken in turbulent boundary layer phenomenology. Published by AIP Publishing. [http://dx.doi.org/10.1063/1.4973243]
\end{abstract}

\section{INTRODUCTION}

The twofold question on whether long-lived vorticitycarrying structures - coherent structures for short—can survive up to higher Reynolds numbers and play an important dynamical role in turbulence, with particular attention to the problems of isotropic and wall-bounded flows, has been for a long time a matter of great interest in the fluid dynamics community. ${ }^{1-7}$

From a modeling perspective, the vorticity field $\vec{\omega}$ of incompressible flows (our focus in this work) can be considered to be a more fundamental observable than the velocity field $\vec{v}$, once the latter can be derived from the former through

$$
v_{i}=-\epsilon_{i j k} \partial^{-2} \partial_{j} \omega_{k},
$$

where, above, $\partial^{-2}$ stands for the inverse Laplacian operator. Of course, Eq. (1.1) is nothing more than the Biot-Savart law in the fluid dynamical context.

One aims, in the so-called "structural formulation of turbulence," to achieve an expressive reduction in the number of degrees of freedom from the introduction of kinematical or dynamical models of coherent structures, the spatial support of strongly correlated vorticity lines. These special vorticity domains are then taken to be the sources of the turbulent velocity field, straightforwardly recovered with the help of Eq. (1.1). It is interesting to point out that while structural modeling is still a very open problem, one finds, within the framework of wavelet compression techniques, strong support for pursuing this direction of research..$^{8-10}$

Among the several types of turbulent flows, the turbulent boundary layer (TBL) is a particularly rich stage for the production and interaction of coherent structures, ${ }^{6}$ like streamwise and hairpin vortices (often bunched in packets), the latter remarkably anticipated several decades ago by Theodorsen ${ }^{11}$ and Townsend. ${ }^{12}$ Due to the variable sizes of these structures, which are directly related to their distances from the wall, as depicted in the attached eddy hypothesis, ${ }^{12,13}$ the TBL turns out to be a dynamical system characterized by strong multiscale couplings.

The pioneering structural approach of Perry and Chong ${ }^{14}$ has underlined in many alternative ways, subsequent investigations of the TBL along the years, ${ }^{15-20}$ devoted to the study of boundary layer phenomena like viscous drag, the existence of enhanced intermittent velocity fluctuations near the wall region, and the crossover between turbulent kinetic energy production and dissipation, all of these being points of potential applied relevance. In spite of its appealing physical picture, the structural approach has been unable, so far, to address in a predictive way a relevant phenomenological framework like the law of the wall. An even more ambitious aim for the structural program would be to provide a foundation for the broadly used Reynolds-averaged phenomenological models (like the $\mathrm{k}$-epsilon model). ${ }^{21,22}$ In these approaches, one has to resort to ad hoc closure assumptions which relate the Reynolds stress 
tensor to the mean properties of the flow. This mathematical object could, as a matter of principle, be derived from the statistical modeling of the energetically most important vortical structures.

While at the present state of knowledge, the aforementioned ideas are still essentially speculative, we show in this work that the structural approach, as based on an accurately validated vortex identification procedure, can offer an interesting insight into the physics of wall bounded flows, if one restricts attention on issues of turbulent isotropization.

A major problem in the structural formulation of turbulence-paradoxically as it may sound-is the ambiguous meaning of the coherent structure concept itself, as long ago emphasized in the seminal papers by Hussain. ${ }^{23,24}$ An operational answer to this question is to define a coherent structure as the compact flow configuration that is obtained, from numerical or experimental data, through the application of some postulated identification algorithm.

Galilean invariant vortex identification methods usually rely on the information encoded in velocity gradients, which tag regions of the flow characterized by "swirling motions" in locally co-moving reference frames. An interesting physical picture underlying the usefulness of velocity gradients in the identification of coherent structures has to do with the empirical fact that they are correlated with the zones of quasi-uniform momentum. ${ }^{25}$ Therefore, velocity gradients are enhanced around the boundaries of such zones, and provide, in this way, "shear envelopes," which are ultimately the reason for the phenomenon of coherent structure persistence, as observed in the dynamics of hairpin vortices. $^{26}$

Most of the discussions on the structural aspects of turbulence adopt Eulerian vortex detection methods like the Qcriterion, ${ }^{27-29}$ the $\Delta$-criterion, ${ }^{30}$ and its closely related swirling strength criterion $\left(\lambda_{c i} \text {-criterion }\right)^{31,32}$ or the $\lambda_{2}$-criterion. ${ }^{33}$ In all of these criteria, a scalar field, derived from the velocity gradient tensor, is used as a "marker" to indicate if a given point in the flow belongs or not to a vortex. Vortices are, therefore, identified as the connected regions mapped by such scalar fields.

Other classes of vortex identification methods shift from the definition of "scalar markers," to representative flow configurations, either by selecting the most energetic ones instantaneously or by retrieving flow patterns by means of statistical averaging procedures. For the sake of completeness, we list below a brief description of five of these approaches.

(i) In the proper orthogonal decomposition, one tries to extract the relevant flow modes that are, on the average, more energetic, by solving associated eigenvalue problems. $^{34}$

(ii) A computer-science inspired approach uses artmap neural networks as a classification tool, in which a self-refining algorithm is used to identify relevant structures. ${ }^{35}$

(iii) Wavelet denoising theory can provide a decomposition of the velocity field on a complete set of orthogonal spatially localized modes, in which the more energetic ones turn out to be associated with coherent structures. ${ }^{8}$ (iv) "Lagrangian coherent structures" can be defined from the investigation, along the pathlines, of the local dynamical system of fluid element motions. 36,37

(v) Conditionally averaged flow configurations, representing coherent structures, can be obtained from a subset of flow realizations that satisfy certain prescribed statistical signatures, a procedure which is closely related to the method of linear stochastic estimation. ${ }^{38,39}$

Even though there are studies which have pointed out the pros and cons of the available vortex identification methods, ${ }^{32,40-44}$ systematic investigations of their limitations are still in order. Commonly noted problems are related to shape distortions of retrieved vortices and the subjective definition of threshold parameters, sometimes necessary to increase the efficiency of the identification algorithms. As we will emphasize in the following, a less obvious (but not less important) difficulty is associated with the effects produced on vortex identification by a shearing environment, as in free shear turbulence, turbulent boundary layers or channel flows.

The velocity gradient-based vortex identification strategies so far addressed in the literature are essentially equivalent, in two-dimensions, to the $\lambda_{c i}$-criterion. This is a key point in our discussion, which relies on a careful study of how the $\lambda_{c i}$-criterion performs for a variety of controlled "synthetic" two-dimensional flow configurations. It turns out that there are serious challenges with the use of the $\lambda_{c i}$-criterion, which have motivated us to introduce an alternative vortex identification prescription, referred to as the vorticity curvature criterion ( $\lambda_{\omega}$-criterion), a vortex identification method entirely based on local properties of the vorticity field.

Our results are centered on the analysis of twodimensional coherent structures, which are important actors, for instance, in the quasigeostrophic approximation for the dynamics of the atmosphere and the ocean (low Rossby number regime, planetary length scales), ${ }^{45}$ in purely twodimensional turbulent systems, ${ }^{46}$ and also in the properties of streamwise/wall normal plane sections of turbulent boundary layer flows, ${ }^{2,47-50}$ which reveal the existence of spanwise vortex tubes. We introduce and study the problem of vortex identification for large ensembles of synthetic two-dimensional vortex systems and subsequently investigate, by means of a turbulent channel flow direct numerical simulation (DNS), the statistical features of boundary layer vortices from the point of view of both the $\lambda_{c i}$ and the $\lambda_{\omega}$ criteria.

This work is organized as follows. To make the paper as self-contained as possible, we provide, in Sec. II, a detailed definition of the $\lambda_{c i}$-criterion, and classify, from the analysis of simple two-dimensional vortex configurations, its main issues. In order to overcome the observed difficulties with the $\lambda_{c i}$-criterion, an essentially threshold-free vortex identification method, the $\lambda_{\omega}$-criterion, is proposed and discussed in Sec. III, which is found to considerably improve vortex detection for most of the problematic cases.

Monte Carlo simulations of synthetic vortex systems are introduced in Sec. IV, as a way to evaluate how the $\lambda_{c i}$-criterion and the $\lambda_{\omega}$-criterion automated algorithms perform for a large number of samples. We find, at this point, poor results for both vortex identification methods for the case of vortices in the presence of a strong background shear. To cope with that, we 
devise a background shear subtraction procedure, meaningful for statistically stationary flows, which points out the better, and reasonably good, performance of the $\lambda_{\omega}$-criterion when compared to the one of the $\lambda_{c i}$-criterion.

We, then, move to the analysis of a more realistic scenario in Sec. V, provided by the numerical simulation of a turbulent channel flow. Having in mind all the issues discussed in the previous sections, it turns out that while the $\lambda_{c i}$-criterion fails to indicate isotropization of small scale turbulent fluctuations in the TBL logarithmic layer, the $\lambda_{\omega}$-criterion can do so, very successfully, which is a remarkable phenomenological result within the context of the structural formulation. We also discuss, in Sec. VI, the extension of the $\lambda_{\omega}$-criterion to the case of fully three-dimensional flows, including some preliminary visualizations for the turbulent channel structures obtained in this way. Finally, in Sec. VII, we summarize our findings and point out the directions of further research.

\section{SWIRLING-STRENGTH ISSUES}

The $\lambda_{c i}$-criterion for vortex identification relies on the analysis of the instantaneous topology of the velocity vector field. ${ }^{31}$ In two dimensions (our main interest in this paper), one wants to single out points of the flow that can be classified either as sources or sinks of streamlines. In more concrete terms, set as $\left(x_{1}, x_{2}\right)=(0,0)$ the position of an arbitrary point in the flow, which has an instantaneous vanishing velocity in the co-moving reference frame. Taking the velocity field to be "frozen," we can write down the linearized equation of motion for a particle that follows the frozen streamlines of the flow in a neighborhood of the origin as

$$
\dot{x}_{i}=A_{i j} x_{j}
$$

where $A_{i j}=\left.\partial_{j} v_{i}\right|_{x=0}$ is the $i, j$ matrix element of the velocity gradient tensor A. It is not difficult to show that the spiraling orbits around the origin (the focus of motion) are necessarily associated with the complex eigenvalues of $\mathbf{A}$. The eigenvalue equation reads

$$
\operatorname{det}\left(\partial_{j} v_{i}-\lambda \delta_{i j}\right)=\lambda^{2}-\lambda \partial_{i} v_{i}+\operatorname{det}\left(\partial_{j} v_{i}\right)=0 .
$$

The "swirling strength" field is the scalar quantity defined as the imaginary part, taken as positive, of the complex eigenvalue $\lambda \equiv \lambda_{c r}+i \lambda_{c i}$. The $\lambda_{c i}$-criterion, thus, postulates that vortex domains are regions of the flow which have non-zero swirling strength. For incompressible two-dimensional flows, things are a bit simpler, once Eq. (2.2) tells us that these regions are the loci of the points where the velocity gradient determinant is positive.

To exemplify the analysis, we illustrate how the $\lambda_{c i}$-criterion works for the prototypical Lamb-Oseen vortex, ${ }^{51}$ which is in fact an important building block in structural studies. ${ }^{18,52-54}$ Let $\epsilon_{i j}$ be the two-dimensional Levi-Civita symbol. The Lamb-Oseen vortex is defined by the divergence free velocity field, with components

$$
v_{i}=\epsilon_{i j} x_{j} F(r)
$$

where

$$
F(r)=\frac{\Gamma}{2 \pi r^{2}}\left(1-e^{-\frac{r^{2}}{r_{c}^{2}}}\right)
$$

Above, $r_{c}$ and $\Gamma$ denote the vortex core radius and its asymptotic circulation, respectively. The velocity gradient determinant can be easily derived as

$$
\begin{aligned}
\operatorname{det}\left(\partial_{j} v_{i}\right) & =F\left[F+r F^{\prime}\right] \\
& =\left(\frac{\Gamma}{2 \pi r^{2}}\right)^{2}\left[1-2 e^{-\frac{r^{2}}{r_{c}^{2}}}+e^{-\frac{2 r^{2}}{r_{c}^{2}}}\left(3-\frac{2 r^{2}}{r_{c}^{2}}\right)\right]
\end{aligned}
$$

and it is shown in Fig. 1 as a function of $r / r_{c}$. The interesting point here is that the velocity gradient determinant is positive only within a finite distance $r \leq \bar{r}$ from the origin, so that the Lamb-Oseen vortex is identified as the disk on the density plot given in the inset of Fig. 1. From Eq. (2.5), we find that $\bar{r}$ and the vorticity flux across the disk, $\bar{\Gamma}$ are related to the corresponding vortex parameters as

$$
r_{c}=\alpha \bar{r} \text { and } \Gamma=\beta \bar{\Gamma},
$$

where in terms of the Lambert $\mathrm{W}$ function ${ }^{55}$

$$
\alpha \equiv \frac{1}{\sqrt{-\frac{1}{2}-W\left(-\frac{1}{2 \sqrt{e}}\right)}} \simeq 0.89
$$

and

$$
\beta=\frac{1}{1-e^{-\alpha^{2}}} \simeq 1.4 .
$$

It is common to assume, as a first approximation, that the connected regions highlighted by the $\lambda_{c i}$-criterion have, even in many-vortex two-dimensional systems, circular shapes, so that the relations given in (2.6) can be used to recover, in an automated fashion, the radius and the circulation parameters of the identified vortices. These same parameters can be obtained, alternatively, but with greater computational cost and comparable accuracy, from fittings, in the spotted regions, of the recorded velocity fields to the Lamb-Oseen pattern, Eqs. (2.3) and (2.4). ${ }^{53,54}$

Serious difficulties can arise in the implementation of the $\lambda_{c i}$-criterion when two or more vortices get close enough to each other, or if they are in the presence of a shearing background. However, there are no comprehensive works in the literature which attempt to define the conditions for the accurate use of this vortex identification method. Therefore, we put forward below, as a necessary stage for an improvement

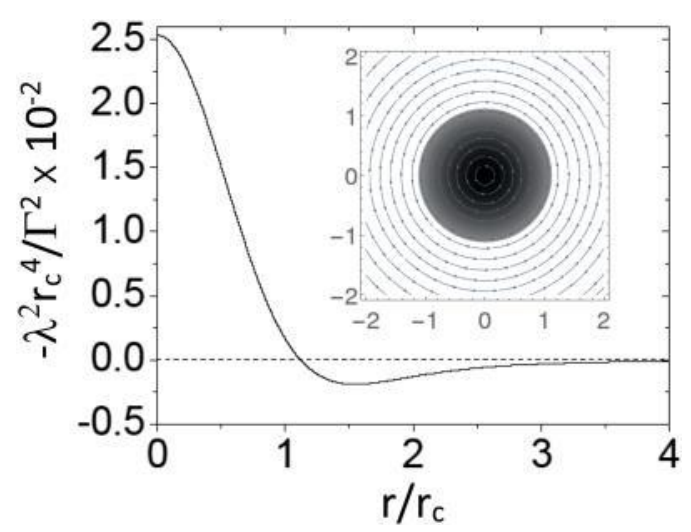

FIG. 1. The dimensionless velocity gradient determinant for the Lamb-Oseen vortex as a function of $r / r_{c}$. Inset: density plot of the swirling strength field and the vortex streamlines (coordinates are given in units of $r_{c}$ ). 
over the $\lambda_{c i}$-criterion, an informal (and not exhaustive) classification of its important problematic issues for the case of two-vortex systems. To render our discussion free of ambiguities, whenever we refer to strict two dimensional vortices throughout the paper, we mean precisely Lamb-Oseen vortices.

\section{A. Vortex shape distortion and coalescence}

As it is shown in Fig. 2(a), the shapes of two vortices get distorted as they approach each other, up to the point where they coalesce into a single vortex structure, as in Fig. 2(b), due to the fact that the streamlines with opposite flow directions can mutually cancel in the region between them. Despite the fact that there are two local swirling strength peaks in the merged region, it is not an obvious task how to disentangle them in practical automated analyses.

In order to solve the vortex merging problem, we could define a threshold parameter $T$ and select the regions of the flow which have $\lambda_{c i}>T$. This can actually break the coalesced structures back to two vortices again, but as a side effect other vortices in the system would be erased from detection. It is also likely that many other coalesced vortices in the flow would not be split in this way. Once there is not a clear prescription on how to define $T$, its choice is essentially subjective, and the threshold solution is far from being a well-established procedure. It should be clear, however, that there should be some room, in principle, for the implementation of iterative thresholding algorithms like the ones used in denoising theory. ${ }^{8}$
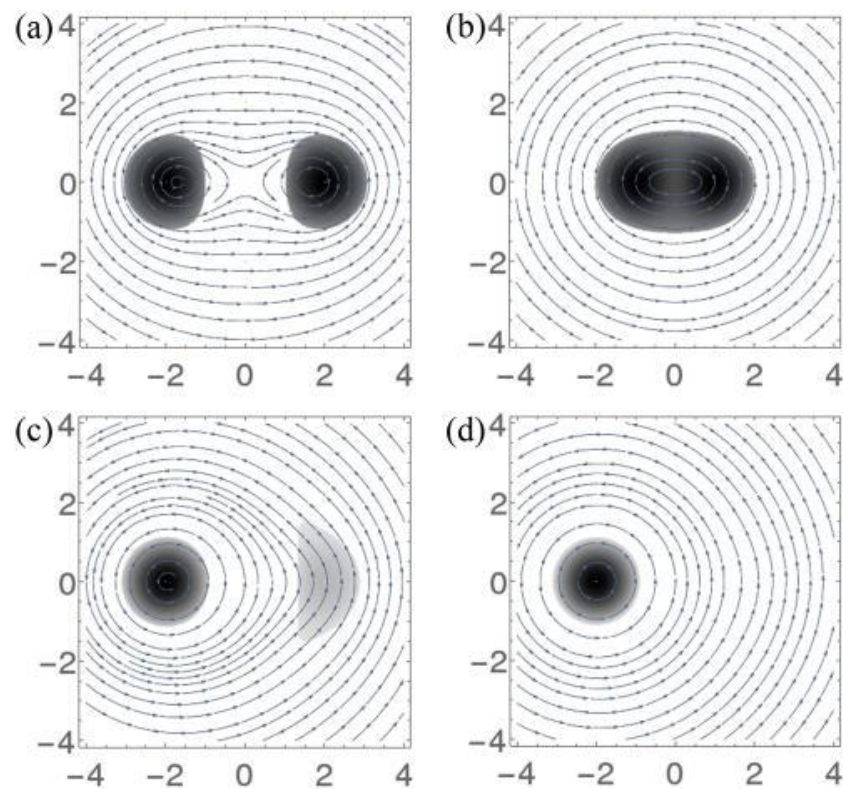

FIG. 2. In all of the four depicted cases, vortex pairs have the same core radius. Coordinates are given in units of $r_{c}$. Let $\Gamma_{L}$ and $\Gamma_{R}$ be the circulations of the left and right vortices, respectively. (a) Shape distortions of two near vortices with $\Gamma_{L}=\Gamma_{R}$; (b) vortex coalescence for a configuration with vortex centers separated by $2 r_{c}$ and $\Gamma_{L}=\Gamma_{R}$; (c) configuration with vortex centers separated by $4 r_{c}$ and $\Gamma_{L}=5 \Gamma_{R}$; (d) the same separation as in (c), but with $\Gamma_{L}=10 \Gamma_{R}$. The right vortex escapes detection by the $\lambda_{c i}$-criterion.

\section{B. Ghost vortices}

Considering two vortices with the same radius, for instance, if one of them has larger circulation, shape distortion is, as expected, more pronounced for the vortex with smaller circulation. Instead of coalescence, however, the weaker vortex can disappear completely from the flow, if it happens to be close enough to the strong one. These situations are depicted in Figs. 2(c) and 2(d).

\section{Background shear effects}

The most dramatic issues on the identification of vortices by means of the $\lambda_{c i}$-criterion are probably the ones associated to background shear effects, which for evident phenomenological reasons, are especially important in wall-bounded flows.

Take, as an illustrative example, the constant background shear with vorticity $\bar{\omega}$, described by the velocity field with components $\left(v_{x}, v_{y}\right)=(-\bar{\omega} y, 0)$, which can be superimposed to the velocity field produced by a vortex or a couple of vortices. Of course, the presence of background shear modifies the velocity gradient determinant. Analogously to Fig. 1, the velocity gradient determinant is plotted in Fig. 3 for $y=0$, as a function of $x / r_{c}$. Differently from the free vortex case, the velocity gradient determinant becomes positive again at some distance from the origin, a fact that is related to the existence of two disconnected and spurious unbounded regions-henceforth referred to as "flaps"-which surround the real vortex, as shown in the inset of Fig. 3. Depending on the intensity and relative sign of the background vorticity, the vortex can disappear and only the flaps remain, or the flaps can coalesce with the vortex, forming a large, unbounded, structure.

In the test situation where we have two Lamb-Oseen vortices with identical circulations in the presence of a constant background shear, the flaps still show up, as can be seen in Figs. 4(a) and 4(b). Furthermore, it turns out that if the background vorticity is opposite to the ones of the two vortices, then, besides the flaps, two spurious vortices appear. More complex patterns arise if additional vortices are superimposed to the background shear flow, once flaps and spurious vortices can also mutually interact.

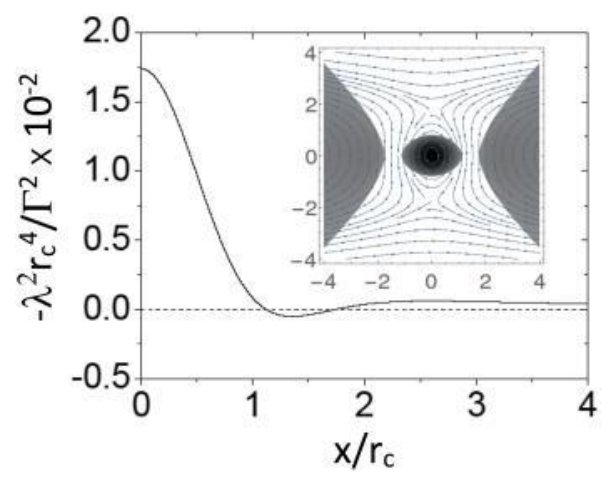

FIG. 3. The dimensionless velocity gradient determinant along the $y=0$ axis, for a vortex of positive circulation $\Gamma$ and radius $r_{c}$ in the presence of a horizontal background shear of negative vorticity $\bar{\omega}=-0.05 \Gamma / r_{c}^{2}$. The $x$ coordinate is given in units of $r_{c}$. Inset: density plot of the swirling strength field for this flow configuration. 

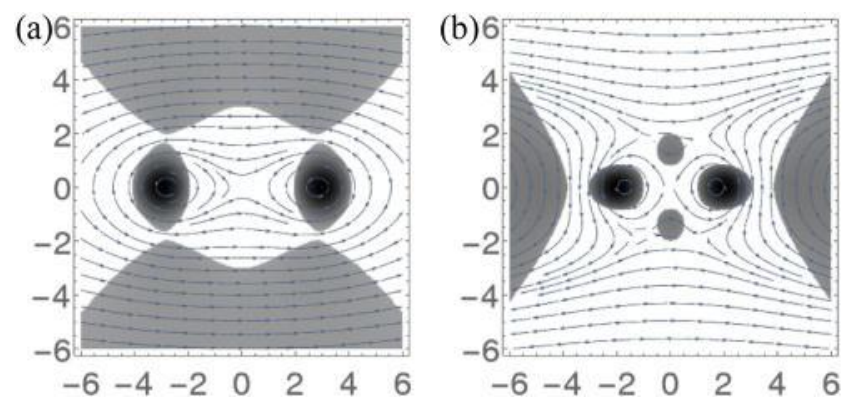

FIG. 4. The background shear is horizontal and both vortices have positive circulation $\Gamma$ and radius $r_{c}$. Coordinates are given in units of $r_{c}$. The background vorticity is $|\bar{\omega}|=0.05 \Gamma / r_{c}^{2}$. (a) Two vortices in a background shear of positive vorticity; (b) two vortices in a background shear of negative vorticity.

\section{Spurious vortices}

Spurious vortices can be misleadingly identified by the $\lambda_{c i}$-criterion in many-vortex configurations. These regions have, in general, relatively small area and circulation, making them, even if sometimes numerous, mostly non-influential to the overall properties of flow, with the exception of counting statistics. Disregarding other aspects of Fig. 4(b), the two vertically aligned and disconnected spots shown, there are examples of spurious vortices generated from the approximation of two real vortices, further enlarged by the presence of background shear, identified in the picture as the two darker disconnected compact regions.

The four general instances discussed above clearly indicate that the analysis of the coherent structures through the use of the $\lambda_{c i}$-criterion, even though meaningful in cases where the vortex density and the vorticity of the background shear are small enough, can lead to inaccurate results, mainly in the investigation of turbulent flows, characterized by strong multiscale intermittent fluctuations of vorticity and strain.

In Sec. III, we put forward an alternative vortex identification method, which has the local vorticity field as its main ingredient and is devised to mitigate the aforementioned deficiencies of the $\lambda_{c i}$-criterion.

\section{VORTICITY CURVATURE CRITERION}

As a key point in understanding the behavior of the $\lambda_{c i}$-criterion in two-dimensional many-vortex systems, it is useful to point out the connection between this criterion and the differential-geometric properties of the stream function $\psi=\psi(\vec{r})$. Note that in a dimensionless system of fluid dynamical units, the Gaussian curvature $K^{56}$ of the stream function graph $(x, y, \psi)$ can be written as

$$
\begin{aligned}
K & =\frac{\partial_{1}^{2} \psi \partial_{2}^{2} \psi-\left(\partial_{1} \partial_{2} \psi\right)^{2}}{1+\left(\partial_{1} \psi\right)^{2}+\left(\partial_{2} \psi\right)^{2}} \\
& =\frac{\partial_{1} v_{1} \partial_{2} v_{2}-\partial_{1} v_{2} \partial_{2} v_{1}}{\left(1+\vec{v}^{2}\right)^{2}}=\frac{\operatorname{det}\left(\partial_{j} v_{i}\right)}{\left(1+\vec{v}^{2}\right)^{2}} .
\end{aligned}
$$

It is clear, thus, from the comparison between (2.2) and (3.2), that in incompressible two-dimensional flows a point belongs to a vortex, according to the $\lambda_{c i}$-criterion, if and only if its stream function graph has positive Gaussian curvature, like a dome.
For a typical vortex, which has two-dimensional vorticity $\omega(\vec{r})$ (a pseudoscalar field) that decays faster than $1 / r$, the streamfunction is asymptotically logarithmic, since

$\psi(\vec{r})=-\partial^{-2} \omega(\vec{r})=\frac{1}{2 \pi} \int d^{2} \vec{r}^{\prime} \log \left(\frac{\left|\vec{r}-\vec{r}^{\prime}\right|}{a}\right) \omega\left(\vec{r}^{\prime}\right)$,

where $a$ is some (unimportant) arbitrary length scale in the flow. The Lamb-Oseen vortex, in particular, is associated to the stream function

$$
\psi=\frac{\Gamma}{4 \pi}\left[\log \left(r^{2} / r_{c}^{2}\right)-\operatorname{Ei}\left(-r^{2} / r_{c}^{2}\right)\right],
$$

where $\operatorname{Ei}(\cdot)$ refers to the Exponential-Integral function, ${ }^{55}$ which is dominated, far from the origin, by the slowly varying logarithmic contribution in Eq. (3.4).

The asymptotic logarithmic profile of the vortex stream function implies that there is strong non-linear superposition effects that affect the curvature of the stream function graph associated to the individual vortices in many-vortex systems. This is the main reason for all of the issues with the implementation of $\lambda_{c i}$-criterion, as discussed in Sec. II. To understand this point in a more detailed way, consider a set of $N$ two-dimensional vortices, placed at positions $\vec{r}_{i}$, which are associated to the respective streamfunctions $\psi_{i}\left(\vec{r}-\vec{r}_{i}\right)$, where $i=1,2, \ldots, N$. The streamfunction at a general position $\vec{r}$ of the flow, is given, therefore, as

$$
\psi(\vec{r})=\sum_{i=1}^{N} \psi_{i}\left(\vec{r}-\vec{r}_{i}\right) .
$$

Since the individual streamfunction fields $\psi_{i}$ have spatial slow logarithmic variations, the above superimposed streamfunction, $\psi(\vec{r})$, can be considerably perturbed by the presence of other vortices in the system.

The ideal setup to deal with vortex identification, thus, would be to base the analysis on the properties of spatially bounded fluid dynamical observables like the vorticity field carried by coherent structures. In two dimensions, the most immediate attempt along these lines would be to work with vorticity level curves, but this is a limited approach, since spurious vortices would proliferate and the subjective choice of thresholds would be unavoidable.

If we insist on vorticity as a fundamental element in a local vortex identification scheme, an interesting heuristic proposal is simply to replace the stream function as it is used in the $\lambda_{c i}$ criterion by the vorticity field. Now, to find vortices, we would look for positive curvature regions of the vorticity graph. This prescription is promising, but the inspection of simple cases suggests that some refinement is still in order.

Consider, for example, four identical vortices which are placed at the vertices of a square. It is not difficult to show that the Gaussian curvature of the vorticity graph is positive at the center of the square, even though there is no vortex there. Without loss of generality, if we take the real vortices to be "bumps" of the vorticity graph (i.e., if they have positive vorticity) then the spurious vortex at the center is a bowl, with idiosyncratic positive vorticity.

In more mathematical terms, we just mean that while $\omega \partial^{2} \omega$ is negative at the square vertices, it changes its sign at the center. This fact is the hint to establish a meaningful vortex identification prescription, the $\lambda_{\omega}$-criterion, which 
relies on the local Gaussian curvature properties of the vorticity graph. To introduce it in detail, we first introduce some notation. Having in mind our two-dimensional context, define, from the vorticity field $\omega(\vec{r})$, the pseudo-velocity field, with Cartesian components

$$
\tilde{v}_{i}(\vec{r}) \equiv \epsilon_{i j} \partial_{j} \omega(\vec{r})
$$

and the pseudo-vorticity field

$$
\tilde{\omega}(\vec{r}) \equiv-\partial^{2} \omega(\vec{r}) .
$$

The streamlines associated to the pseudo-velocity field for the case of a single Lamb-Oseen vortex are qualitatively the same as the ones derived for the physical velocity field, so that they still represent a swirling motion. The main advantage in the use of above definitions is that while they do not spoil the physical meaning of what we consider to be a standard vortex, they are mathematical functions with more interesting local properties, like a fast Gaussian decay as the radial distance from the vortex center increases.

We can also write down the determinant of the pseudovelocity gradient tensor as

$$
\operatorname{det}\left(\partial_{j} \tilde{v}_{i}\right) \equiv-\tilde{\lambda}^{2} .
$$

Taking the imaginary part of $\tilde{\lambda}$ as positive, consider the scalar field

$$
\lambda_{\omega} \equiv \Theta\left(-\omega \partial^{2} \omega\right) \operatorname{Im} \tilde{\lambda}=\Theta(\omega \tilde{\omega}) \operatorname{Im} \tilde{\lambda},
$$

where $\Theta(\omega \tilde{\omega})$ is the Heaviside filtering function that is expected to vanish for spurious vortices, like the one discussed in the preceding four-vortex example. Vortices are then identified by the $\lambda_{\omega}$-criterion as the connected regions of the flow where $\lambda_{\omega} \neq 0$.

Comparing the $\lambda_{\omega}$-criterion to the $\lambda_{c i}$-criterion, we note that the essential advantage of the former is that it depends locally on the vorticity field, which has sharp peaks and rapidly decaying tails for general vortices. The $\lambda_{c i}$-criterion, on its turn, is related to the curvature properties of the stream function graph, which has much broader peaks and tails, and may lead to poor vortex identification resolution.

The $\lambda_{\omega}$-criterion can be classified as a higher order derivative vortex identification scheme, since it depends on the evaluation of third order derivatives of the velocity field (in contrast to the $\lambda_{c i}$-criterion, which is defined in terms of first order derivatives). Two decades ago this fact would be probably a main objection to its practical use. However, taking into account the present status of optical measurement techniques such as particle image velocimetry and the fast increasing computational power of direct numerical simulations, there is an open avenue for the investigation of high-order derivative vortex identification methods. A point of great relevance here is that the $\lambda_{\omega}$-criterion works efficiently even without the imposition of subjective threshold parameters. This brings considerable simplification in the implementation of automated analyses of many-vortex configurations.

We re-examine, now under the light of the $\lambda_{\omega}$-criterion, the relevant vortex identification issues presented in Sec. II. The results are schematically depicted in Fig. 5.

Without background shear, the $\lambda_{\omega}$-criterion has, clearly, higher resolution than the standard $\lambda_{c i}$-criterion, since it is able to split coalesced vortices (Fig. 5(a)) that would otherwise
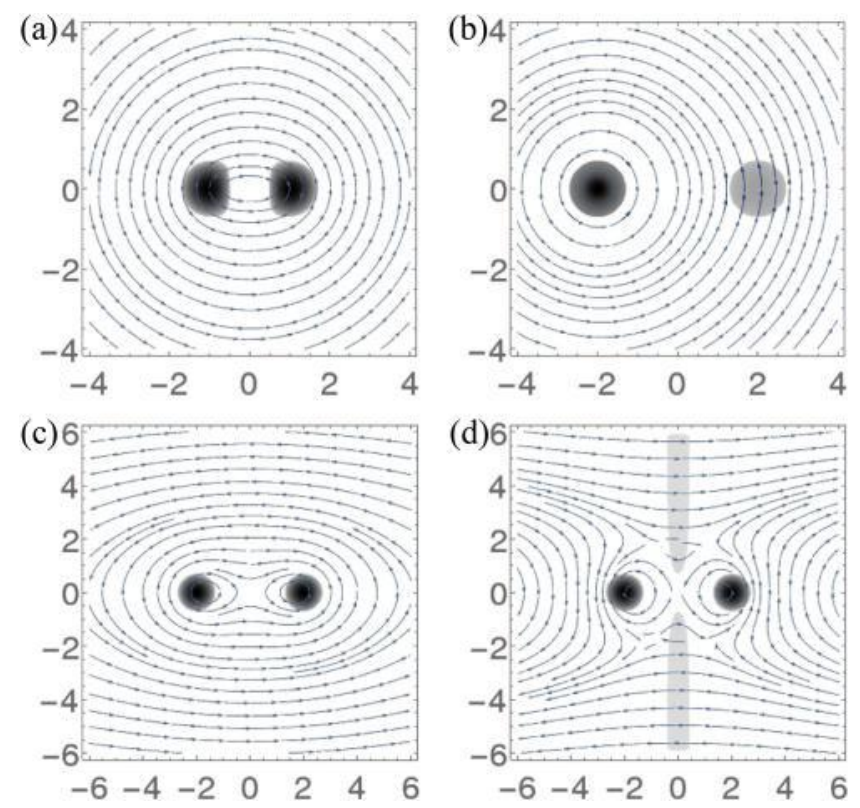

FIG. 5. In (a)-(d), the respective vortex configurations previously studied by means of the $\lambda_{c i}$-criterion in Figs. 2(b), 2(d), 4(a), and 4(b) are now reanalysed, taking the $\lambda_{\omega}$-criterion as the vortex detection tool.

be counted as one, and to recover ghost vortices (Fig. 5(b)). With constant background shear, we also find improvements: the vortex shape distortion is considerably reduced and the large, unbounded flaps are completely eliminated (Figs. 5(c) and 5(d)). However, as it can be seen in Fig. 5(d), there is a couple of relatively small $\lambda_{\omega}$ spurious regions in the form of vertical stripes, produced for the case where the two vortices have vorticity opposite to the one of the background. This undesirable effect is due to the specific form of the filtering function $\Theta(\omega \tilde{\omega})$. If a background with constant vorticity $\bar{\omega}$ is added to the vorticity field $\omega$, the filtering function can be written as $\Theta((\omega+\bar{\omega}) \tilde{\omega})$. Therefore, if $\bar{\omega}$ and $\omega$ have opposite signs and $|\bar{\omega}|>|\omega|$, the filtering function may, as a side effect, introduce errors or even hamper the identification of a true vortex. We will have more to say about this issue in Sec. IV.

In order to illustrate the crucial importance of the filtering function and the general improvement gained with the $\lambda_{\omega}$-criterion over the $\lambda_{c i}$-criterion, we show in Fig. 6 the analysis of a sample of 20 Lamb-Oseen vortices with varying radii and circulations, which are randomly distributed in a square domain. While the use of the $\lambda_{c i}$-criterion is unable to avoid the merging of two of the vortices and the disappearance of another one, all of the vortices are recovered with the use of $\lambda_{\omega}$-criterion, which approximately preserves their original circular shapes.

If the filtering function were not used, many spurious regions would remain, as evidently pointed out in Fig. 6(c). One notices that a few spurious vortices have survived the screening of the $\lambda_{\omega}$ criterion. We have to keep in mind, for proper applications of the $\lambda_{\omega}$-criterion, that although leading to improvements, it is not free of errors, in the sense that probably any meaningful vortex identification method will eventually break in the analysis of extreme (hopefully unrealistic) flow conditions. 

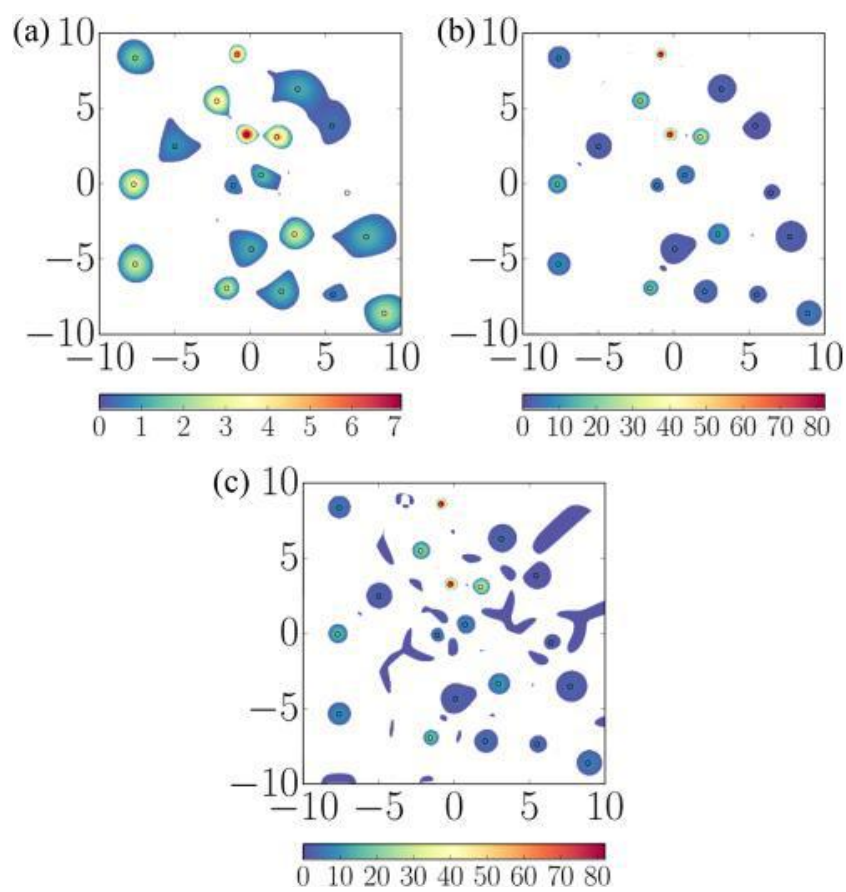

FIG. 6. Small open circles indicate the positions of 20 randomly distributed vortices. (a) Vortex detection via the $\lambda_{c i}$-criterion. The phenomena of vortex coalescence and vortex erasing take place, respectively, in the first and fourth quadrants of the domain; (b) vortex detection via the filtered $\lambda_{\omega}$-criterion, where all of the original vortices have been identified; (c) inaccurate vortex detection via the unfiltered $\lambda_{\omega}$-criterion. The color bars represent the $\lambda_{c i}$ and $\lambda_{\omega}$ fields in arbitrary units.

At this point, it is interesting to briefly discuss the relevance of the Lamb-Oseen vortex as a standard of analysis. The Burgers vortex ${ }^{51}$ could be an alternative, having in mind that it is perhaps a more relevant structure for general turbulence modeling, as it has been suggested from turbulent wind tunnel experiments, ${ }^{57}$ and from the fact that it can play an important role in the theoretical understanding of intermittency in homogeneous and isotropic turbulence. ${ }^{58}$ However, it turns out that if we are actually interested to focus on the performance of vortex identification methods, more than on modeling issues, the Lamb-Oseen vortex is by far the simpler and more convenient choice, leading to equivalent conclusions. More specifically, while the Burgers vortex is defined from four independent parameters (two strain rate eigenvalues, the asymptotic circulation, and its core radius), the Lamb-Oseen vortex is completely determined by its asymptotic circulation and core radius parameters. It is not difficult to show that while the variations of the two extra-parameters for the Burgers vortex are rigorously harmless in the context of the $\lambda_{\omega}$-criterion, they may affect the performance of the $\lambda_{c i}$-criterion in unwanted ways, due to the presence of additional shearing.

So far, all of our arguments have been based on the inspection of a few representative analytical vortex configurations. Of course, more is needed to validate the $\lambda_{\omega}$-criterion as a reliable tool. This is our next step, to be carried out with the help of extensive Monte Carlo simulations, where we consider, instead, discretized velocity derivatives for the analysis of large ensembles of synthetic many-vortex systems.

\section{MONTE CARLO STUDY}

To address a comparative study of accuracy for the $\lambda_{\omega}$ and the $\lambda_{c i}$ criteria, we run Monte Carlo tests for large ensembles, where in each sample vortices are randomly distributed over the area of a square domain. The velocity field over a discretized grid is recorded and the two vortex identification criteria are applied to investigate how they perform in detecting and also in recovering the properties (circulation, radius, and position) of the original vortices.

In all of the synthetic samples, evaluations of the velocity gradient, pseudo-velocity, and pseudo-velocity gradient have been done with five-point weighted finite differences, which in the worst situations (the ones involving three derivatives of the velocity field) have precision of $O\left(\delta^{2}\right)$ in the grid spacing $\delta$. Integrations rely on bilinear interpolations, which are also precise to $O\left(\delta^{2}\right)$. The connected regions where vortices are detected are individualized in the grid with the use of a connected component labeling algorithm. ${ }^{59}$ For each connected region $R_{k}(k=1,2, \ldots)$ we compute

$$
\begin{gathered}
A_{k} \equiv \pi \bar{r}^{2}=\int_{R_{k}} d^{2} \vec{r}, \\
\bar{\Gamma}_{k}=\int_{R_{k}} \omega(\vec{r}) d^{2} \vec{r}, \\
\left(x_{k}, y_{k}\right) \equiv \frac{\int_{R_{k}}(x, y) \omega^{2}(\vec{r}) d^{2} \vec{r}}{\int_{R_{k}} \omega^{2}(\vec{r}) d^{2} \vec{r}} .
\end{gathered}
$$

Eqs. (4.1) and (4.2) allow us to infer, respectively, with the help of Eq. (2.6), the real radius $r_{k}$ and circulation $\Gamma_{k}$ vortex parameters. While for the $\lambda_{c i}$-criterion, $\alpha$ and $\beta$ are already known from Eqs. (2.7) and (2.8), a similar and straightforward analysis for the $\lambda_{\omega}$-criterion yields the analogous pair of parameters $(\alpha, \beta)=(\sqrt{2}, 1 /(1-1 / \sqrt{e})) \simeq(1.41,2.54)$. Additionally, Eq. (4.3) gives the "center of enstrophy" coordinates for the position of the identified vortex. The $\alpha$ parameter for vortex core radius conversion is, in the $\lambda_{\omega}$-criterion, about 1.6 times greater than the one for the $\lambda_{c i}$-criterion. This is a casual but nevertheless very helpful fact, since it improves the resolution of the detected structures, as it could have already been noticed from the former's section results.

We have worked, for a set of flow configurations of interest, with $N=10^{5}$ Monte Carlo samples, each one containing $N_{v}=20$ randomly distributed vortices, on a $[-9,9]^{2}$ square (arbitrary length scale). The velocity field is exactly defined at the sites of a $N_{x} \times N_{y}=200^{2}$ grid, which models the square box $[-10,10]^{2}$. When sampled, vortex centers are always separated by distances greater than 1.2 times the sum of their radii. ${ }^{60}$ Circulations and vortex radii are sampled with uniform random distribution in the domains given, respectively, by $1 \leq|\Gamma| \leq 20$ (or $-20 \leq \Gamma \leq-1$ ) and $0.5 \leq r_{c} \leq 1.5$.

As a way to get rid of spurious vortices, we furthermore prescribe that $R_{k}$ is accepted as vortex only if $\left|\Gamma_{k}\right| \geq \Gamma_{0}$, for some small circulation scale $\Gamma_{0}$. Note that this cutoff prescription is conceptually distinct from the imposition of a threshold, where the main worry is not exactly on the existence of spurious vortices as individual objects, but on specific-noise 
TABLE I. General definitions for the Monte Carlo simulations of the synthetic many-vortex two-dimensional systems.

\begin{tabular}{ll}
\hline \hline Number of samples & $N=10^{5}$ \\
Number of vortices/sample & $N_{v}=20$ \\
System's dimensions & $\left(L_{x}, L_{y}\right)=(20,20)$ \\
Vortex positions & $-9 \leq x, y \leq 9$ \\
Grid size & $200 \times 200$ \\
Vortex circulations & $\Gamma \in \pm[1,20]$ \\
Vortex core radii & $r_{c} \in[0.5,1.5]$ \\
Acceptance cutoff & $\Gamma_{0}=0.5$ \\
Vortex pair separation & $d_{i j}>1.2 \times\left(r_{c i}+r_{c j}\right)$ \\
\hline \hline
\end{tabular}

contaminated-regions of the flow. The circulation cutoff for vortex acceptance is defined as $\Gamma_{0}=0.5$. The Monte Carlo simulation definitions are summarized in Table I.

Motivated by the distribution of spanwise vortices observed in streamwise/wall normal planes of turbulent boundary layers, ${ }^{2,47-49,52-54}$ we have considered, in our Monte Carlo simulations, five distinct flow patterns, denoted by Latin capital letters from A to E, described in Table II.

To define the weak and strong shear regimes referred to in Table II, observe, as it can be derived from (2.2), that a vortex with peak vorticity $\omega_{p}$ disappears from swirling strength detection if the vorticity of the background shear is $|\bar{\omega}|>\left|\omega_{p}\right| / 2$, with $-\bar{\omega} \omega_{p}<0$. Recalling that for a Lamb-Oseen vortex, $\omega_{p}=\Gamma / \pi r_{c}^{2}$, and that in our Monte Carlo samples, $|\Gamma| \leq 20$ and $\left|r_{c}-1\right| \leq 0.5$, we take, as representative parameters, $\Gamma=10$ and $r_{c}=1$, which lead to $\omega_{p} / 2 \simeq 1.6$. Weak and strong regimes are then defined as the ones which have background velocity field components given, respectively, by $\left(v_{x}, v_{y}\right)=(0.35 y, 0)$ and $\left(v_{x}, v_{y}\right)=(1.6 y, 0)$. Note that for flow patterns with either weak or strong background shear, the background vorticity is negative.

In the following, we organize the large lists of input and output vortex parameters (circulation, radius, and position) in the form of histograms that indicate how the $\lambda_{c i}$ and $\lambda_{\omega}$ vortex identification criteria perform in the automated analysis of Monte Carlo ensembles.

Results for the flow pattern A are given in Fig. 7. The $\lambda_{\omega^{-}}$ criterion has an excellent performance, while the $\lambda_{c i}$-criterion is mainly affected by vortex coalescence, which explains why the counting is reduced for the larger vortices and why so many non-existent structures with circulation $|\Gamma|>20$ have been artificially produced. One can note, from Figs. 7(c) and 7(d) that there are boundary effects in the distribution of vortices. This is actually due to the fact that by definition they "avoid each other" in the bounded domain. The same feature is observed in all of the other flow patterns.

TABLE II. The five flow patterns considered in our Monte Carlo simulations.

\begin{tabular}{lcl}
\hline \hline Flow pattern & Vortex circulations & Background shear \\
\hline A & $1 \leq|\Gamma| \leq 20$ & No background \\
B & $1 \leq|\Gamma| \leq 20$ & Weak \\
C & $1 \leq|\Gamma| \leq 20$ & Strong \\
D & $-20 \leq \Gamma \leq-1$ & No background \\
E & $-20 \leq \Gamma \leq-1$ & Strong \\
\hline \hline
\end{tabular}
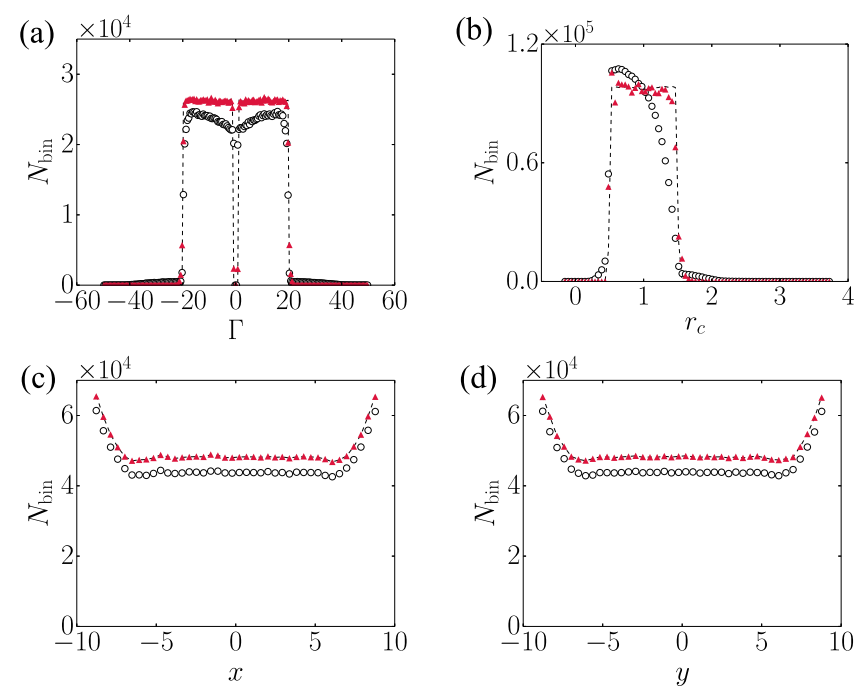

FIG. 7. Flow pattern A. Histograms for performance comparison between the $\lambda_{\omega}$-criterion (triangles) and the $\lambda_{c i}$-criterion (circles), in the evaluation of vortex parameters. (a) Circulations; (b) radii; (c) $x$ coordinates; (d) $y$ coordinates. The dashed lines are the histograms for the input data.

For the flow patterns $\mathrm{B}$ and $\mathrm{C}$, which have weak and strong background shear, respectively, the related histograms are given in Figs. 8 and 9. In the flow pattern B, as shown in Fig. 8, the $\lambda_{c i}$-criterion yields a small and uniform suppression of vortices in the samples, but the circulation and radius countings are actually close to the ones found for the flow pattern A. The $\lambda_{\omega}$-criterion is still the better choice, despite the fact that vortex counting is strongly affected by the addition of spurious vortices of small circulation and artificial structures like the stripes previously observed in Fig. 5(d). Actually, as we will see in a moment, the $\lambda_{\omega}$-criterion is able to capture the input vortices in this case, which are more precisely counted when background shearing effects are removed.

Driving our attention now to the flow pattern C, Fig. 9 tells us that both the $\lambda_{c i}$ and the $\lambda_{\omega}$ criteria perform badly. It turns out that strong external shearing introduces, in general, relevant effects in vortex identification that demand improvement.
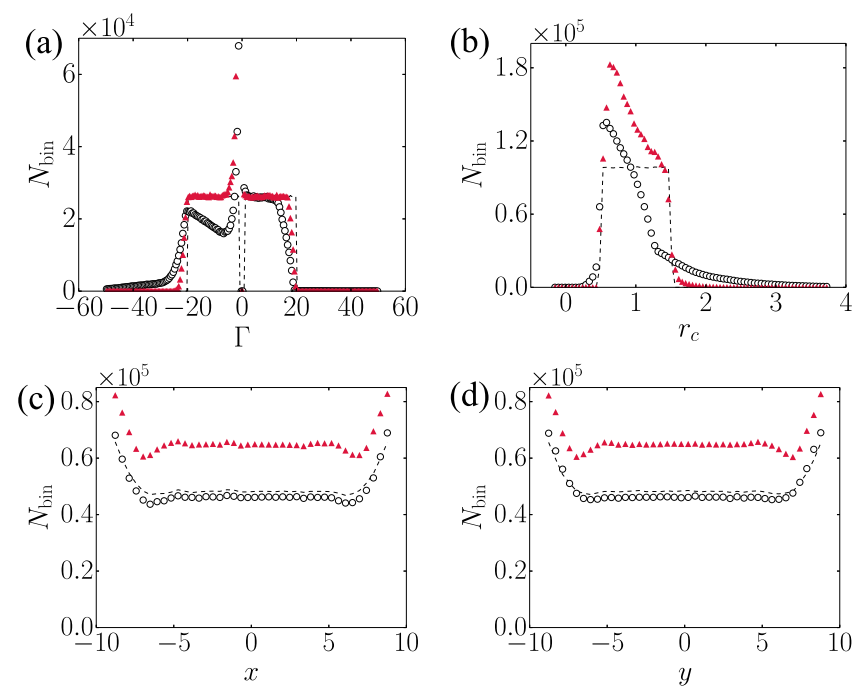

FIG. 8. Flow pattern B. All the rest as in the caption of Fig. 7. 

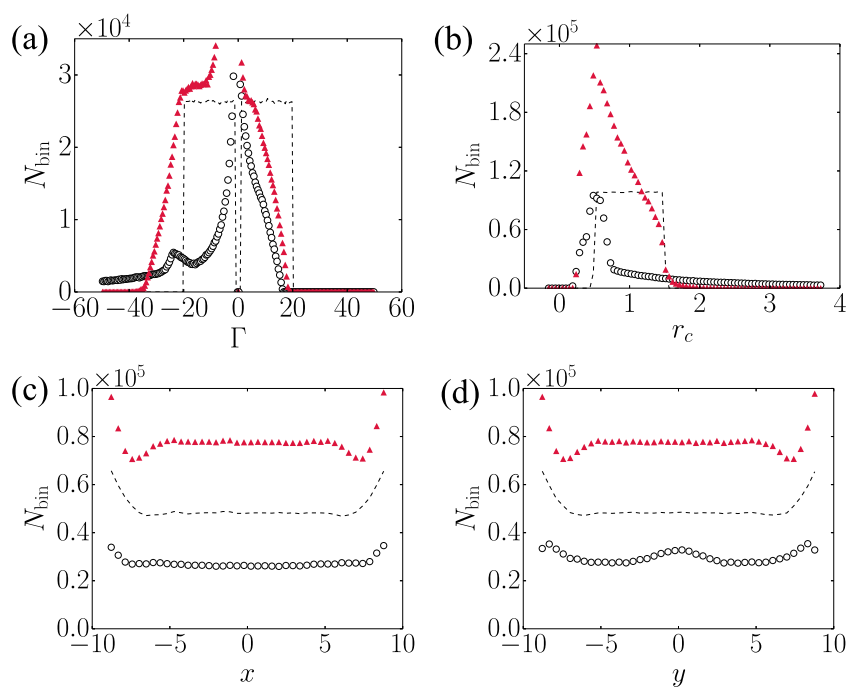

FIG. 9. Flow pattern C. All the rest as in the caption of Fig. 7.

The visualization of a typical Monte Carlo sample of the flow pattern $\mathrm{C}$ is given in Fig. 10, where we see, as a dominant effect, coalescence percolation of flaps and vortices in the application of the $\lambda_{c i}$-criterion. On the other hand, the image associated to the $\lambda_{\omega}$-criterion looks qualitatively different, and although most of the input vortices have been retrieved from the sample, they are surrounded by several spurious structures that can spoil the histograms, like the ones we consider here.

In order to deal with the shortcomings associated with shearing/vorticity backgrounds, we put forward an improved computational strategy, based on the subtraction of the background velocity field, sample by sample, from individual velocity field realizations. This is, of course, nothing more than the method of Reynolds decomposition, which, actually, has been already employed in the previous studies of coherent structure identification, as in Ref. 61. The idea, thus, is to revisit our previous analyses, by just replacing the original velocity field components $v_{i}(\vec{r})$ by its fluctuations over the background, that is,

$$
\delta v_{i}(\vec{r})=v_{i}(\vec{r})-\left\langle v_{i}(\vec{r})\right\rangle,
$$

where $\left\langle v_{i}(\vec{r})\right\rangle$ stands for the expectation value of the velocity field taken over the ensemble of configurations. Furthermore,

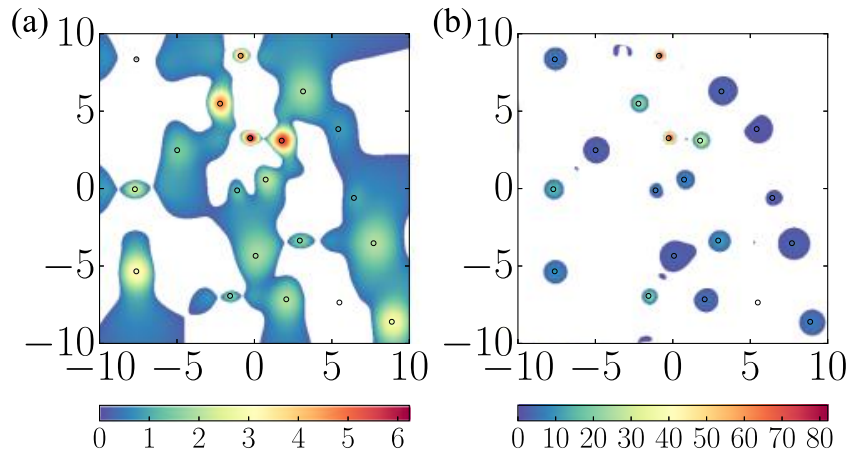

FIG. 10. A sample of 20 vortices-the same as in Fig. 6, now in the presence of strong background shear (flow pattern C), investigated through the (a) swirling strength and (b) the vorticity curvature fields. The color bars represent the $\lambda_{c i}$ and $\lambda_{\omega}$ fields in arbitrary units.
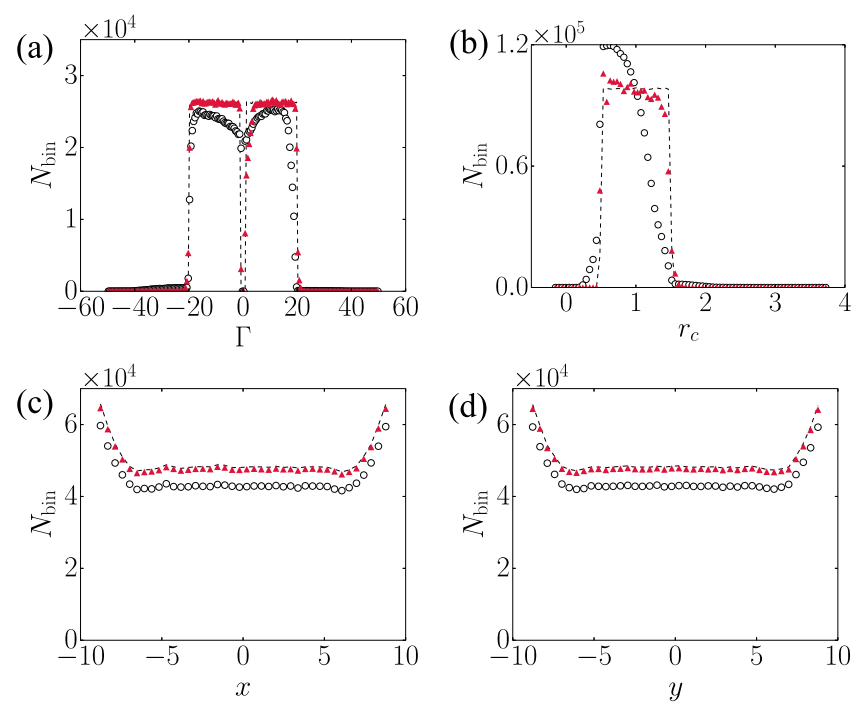

FIG. 11. Analysis of the flow pattern B, with background subtraction. All the rest as in the caption of Fig. 7.

as an important prescription, in order to avoid additional spurious effects, we assign a given point in the flow to a vortex if it is detected in the vortex identification screening carried out with and without the background subtraction procedure.

We compare, in the next six sets of histograms, the performance of the $\lambda_{c i}$ and the $\lambda_{\omega}$ criteria, both with background subtraction procedure for the flow patterns $\mathrm{B}$ and $\mathrm{C}$, while analogous comparisons are done for the flow patterns $\mathrm{D}$ and $\mathrm{E}$, with and without background subtraction. We do not report here the additional background subtraction analysis of the flow pattern A, since (as expected) we find that both criteria work again as in Fig. 7, due to the fact that the balanced mixing of vortices with positive and negative circulations produces a very small background.

The weak shear case, flow pattern B, is given in Fig. 11, where both the $\lambda_{c i}$ and $\lambda_{\omega}$ criteria are noted to improve in their performances, with a clear advantage for the latter.
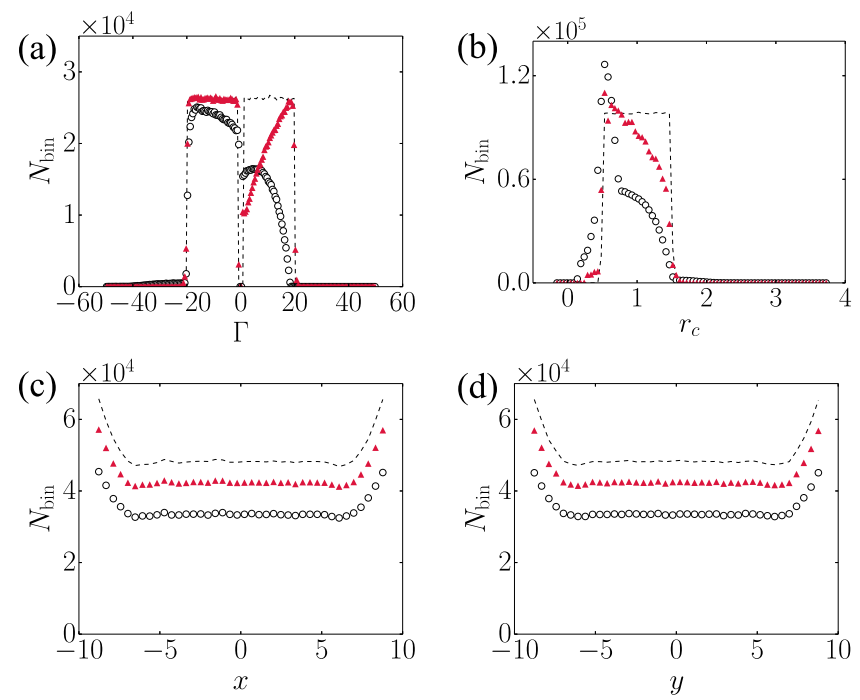

FIG. 12. Analysis of the flow pattern C, with background subtraction. All the rest as in the caption of Fig. 7. 

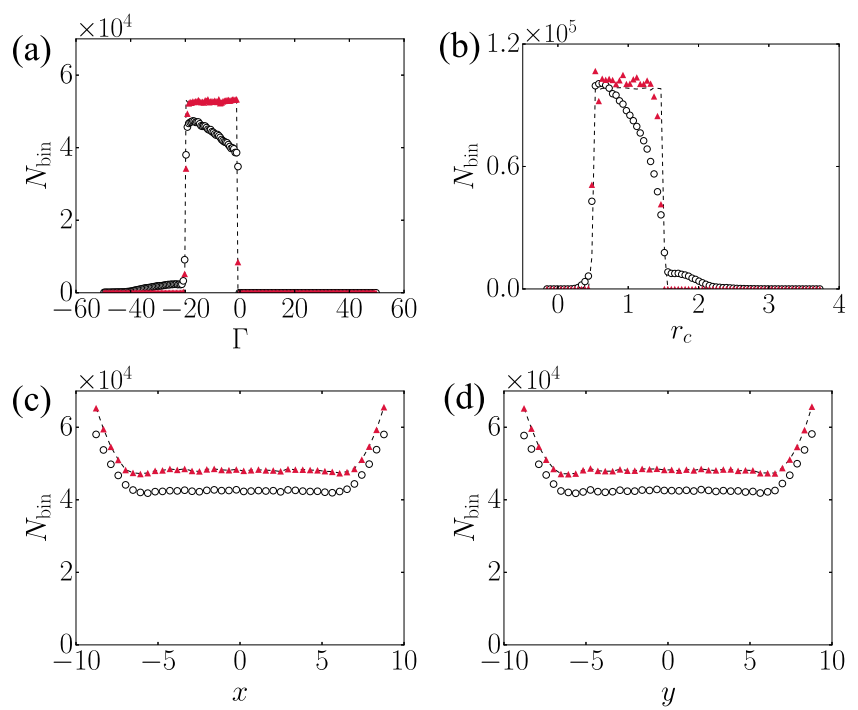

FIG. 13. Analysis of the flow pattern D, without background subtraction. All the rest as in the caption of Fig. 7.

For the flow pattern $\mathrm{C}$, we conclude, from Figs. 9 and 12 , that the background subtraction procedure considerably improves the performance of the $\lambda_{\omega}$-criterion, which now becomes valid as a method of vortex identification. Its only residual deficiency is the suppression of vortices which have relatively large radii and small positive circulations. This is, very clearly, a side effect of the Heaviside filtering function, which erases positive-circulation vortices that are completely "submerged" in the negative vorticity background.

As a way to loosely mimic some of the turbulence boundary layer characteristics found in streamwise/wall normal planes, where the background vorticity has the same sign as most of the viscous layer vortices, ${ }^{2,49,53,54}$ we have devised the flow regimes $\mathrm{D}$ and $\mathrm{E}$. Note that in the flow pattern $\mathrm{D}$, there is no external background, $\bar{\omega}=0$, but there is an essentially uniform negative vorticity background produced by the manyvortex system because $\left\langle v_{i}(\vec{r})\right\rangle \neq 0$. Curiously, as it can be seen
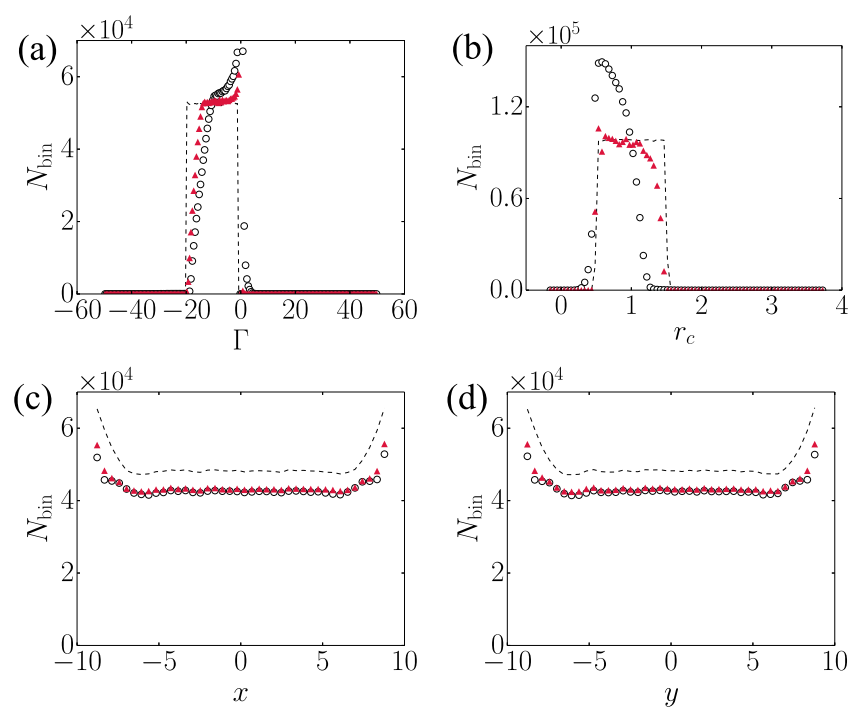

FIG. 14. Analysis of the flow pattern D, with background subtraction. All the rest as in the caption of Fig. 7.
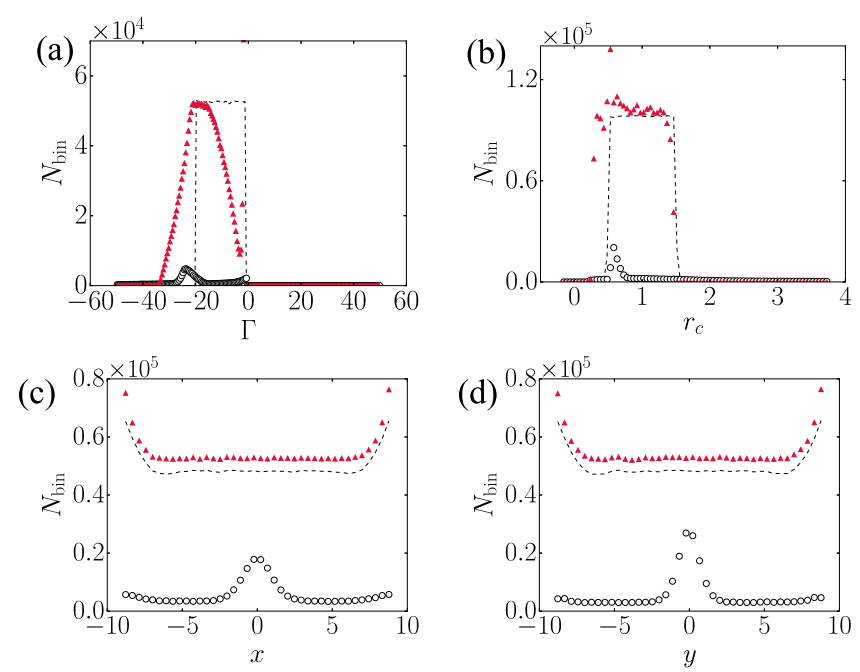

FIG. 15. Analysis of the flow pattern E, without background subtraction. All the rest as in the caption of Fig. 7.

from Figs. 13 and 14 , the $\lambda_{\omega}$-criterion is acceptable in both cases, but it works a bit better, for the flow pattern $\mathrm{D}$, if the background was not subtracted. This has to do, this time, with the existence of vortices that are placed in regions of the flow where the local vorticity background is momentarily greater, due to the effect of fluctuations, than the mean self-induced vorticity background.

For the strong background case, flow pattern E, it turns out, as indicated from Figs. 15 and 16, that the background subtraction procedure leads to an improvement, mainly in recovering circulation statistics, which brings the quality of vortex identification back to the reasonably good standards observed in the analysis of the flow pattern D.

The above benchmarking Monte Carlo study shows that the $\lambda_{\omega}$-criterion, enhanced by the background subtraction procedure, provides an appropriate identification prescription for the investigation of two-dimensional vortex systems. With the confidence acquired from the numerical experiments carried out with synthetic samples, we focus now on the analysis of a more realistic flow situation.
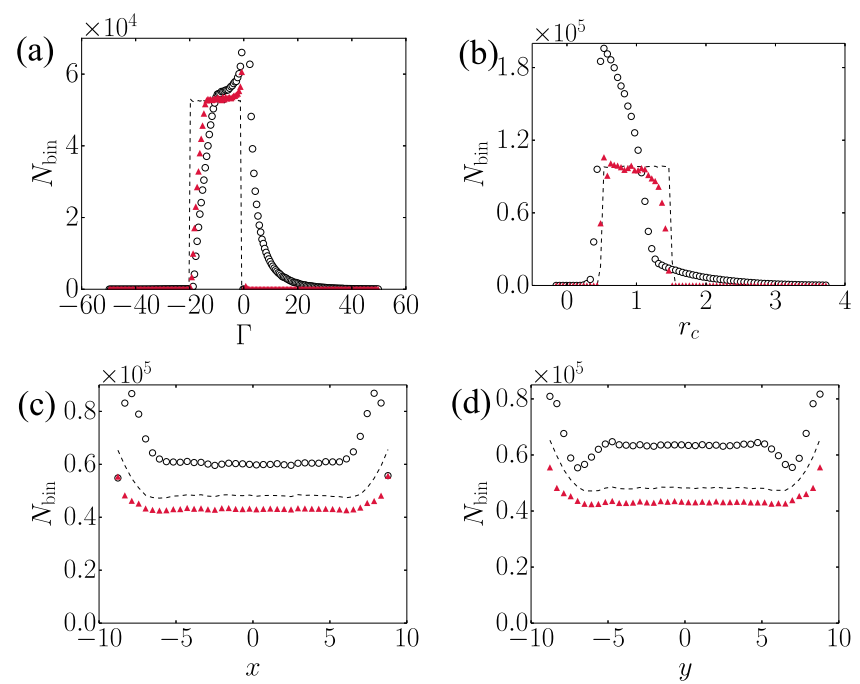

FIG. 16. Analysis of the flow pattern E, with background subtraction. All the rest as in the caption of Fig. 7. 


\section{APPLICATION TO A TURBULENT CHANNEL FLOW}

Cross sections of spanwise vortices, interpreted as heads of hairpin vortices, have been usually observed in streamwise/wall normal plane sections of wall-bounded flows. ${ }^{2,47-50,52-54}$ We have investigated the statistical properties of such two-dimensional vortex flow patterns by means of the $\lambda_{c i}$ and the $\lambda_{\omega}$ criteria, for a turbulent channel flow DNS.

The turbulent channel flow simulation has friction Reynolds number $R e_{\tau} \simeq 395$ and setup parameters described in Table III. We follow here the simulation guidelines put forward by Kim, Moin, and Moser. ${ }^{62}$ The streamwise, normal to the wall, and spanwise coordinates are, respectively, $x, y$, and $z$; periodic boundary conditions are imposed along the streamwise and spanwise directions; the grid is not uniform, with enhanced resolution near the walls, so that the viscous sublayer can be resolved with approximately one viscous length per lattice spacing. The simulation has been validated by standard tests, like the reproduction of the law of the wall and of statistical moments.

We have recorded, at every ten time steps in the turbulent stationary regime, the projection of the velocity field of three parallel streamwise/wall normal planes $z=0, z=\pi / 3$, and $z=2 \pi / 3$. The ensemble defined in this way has a total number of 5268 flow configuration snapshots, which are, then, studied as two-dimensional velocity fields.

We show, in Fig. 17, vortex identification images for one representative snapshot, analysed in three different ways. Figs. 17(a) and 17(b) give the results obtained from the application of the $\lambda_{c i}$-criterion without and with the use of the background subtraction procedure, respectively. Fig. 17(c) is the analogous result associated to the use of the $\lambda_{\omega}$-criterion with background subtraction; no circulation cutoff has been used in the identification of vortices.

There are expressive qualitative differences between the two images produced by the $\lambda_{c i}$-criterion, for regions which are closer to the wall, where shear effects become more relevant. The $\lambda_{\omega}$-criterion leads, on the other hand, to a much better vortex resolution, but the background subtraction procedure does not lead, in visual terms, to expressive modifications-that is why we have not shown the picture associated to the application of the $\lambda_{\omega}$-criterion without background subtraction. This, in fact, suggests that the flow takes place in weak background shear conditions. There are, however, small but meaningful improvements from the use of the background subtraction procedure that become evident only through histogram analysis, as we will show below.

As a practical remark to be emphasized here, we note that as it is a higher order derivative method, the $\lambda_{\omega}$-criterion is related to the identification fields that typically fluctuate over a much wider range of values than the ones associated to the

TABLE III. Parameters for the DNS of a turbulent channel flow.

\begin{tabular}{ll}
\hline \hline System's dimensions & $\left(L_{x}, L_{y}, L_{z}\right)=(2 \pi, 2, \pi)$ \\
Grid size & $256 \times 192 \times 192$ \\
Kinematic viscosity & $v \simeq 8.6 \times 10^{-4}$ \\
Kinematic pressure gradient & $d P / d x=0.11$ \\
Simulation time step & $\Delta t=1.2 \times 10^{-3}$ \\
\hline
\end{tabular}

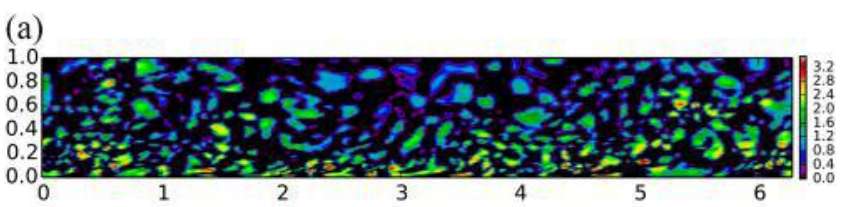

(b)

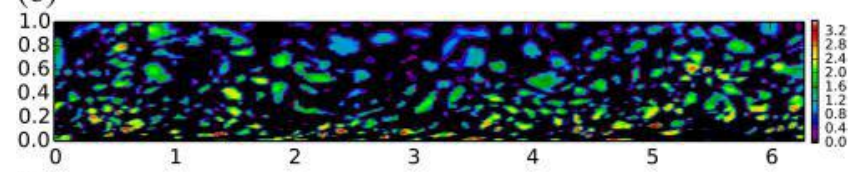

(c)

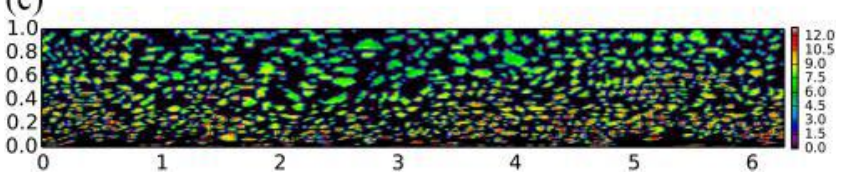

FIG. 17. Density plots of the $\lambda_{c i}$ [figures (a) and (b)] and the $\lambda_{\omega}$ [figure (c)] fields in a streamwise/wall normal plane for the DNS of a turbulent channel flow, for all the channel extension and from the bottom wall up to the midchannel height. No threshold is used in the vortex identification analyses. The background subtraction procedure is implemented only in figures (b) and (c). The color bars represent the $\lambda_{c i}$ and the $\lambda_{\omega}$ fields in linear and logarithmic scales, respectively.

$\lambda_{c i}$-criterion. This justifies our use of the logarithmic scale in the elaboration of the image given in Fig. 17(c). Fixing attention on the $\lambda_{\omega}$-criterion, the natural application of the logarithmic scale implies, furthermore, that an optional use of thresholds is somewhat delicate for the case of turbulent (intermittent) flows: in fact, if the threshold is defined, for instance, as $20 \%$ of the maximum value of the logarithm of the $\lambda_{\omega}$ field, then its effects are likely to be irrelevant, since only structures with very low kinetic energy would be discarded; alternatively, if an analogous definition of the threshold is given in a linear scale, it is not difficult to see that almost all of the vortex structures would be erased in this way.

A closer look at the structures identified by the $\lambda_{\omega}$-criterion is given in Fig. 18, where we plot their contours and the surrounding streamlines, computed for the velocity field fluctuations around their mean values. The streamwise

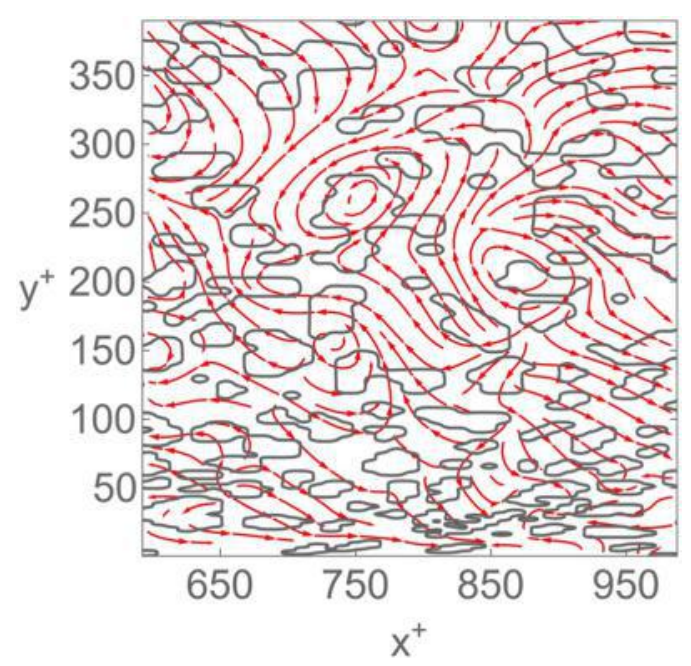

FIG. 18. Streamlines (red lines) for the velocity fluctuations around the mean flow and the closed contours (grey lines) of vortices identified through the vorticity curvature criterion, in the region of wall units $0 \leq y^{+} \leq 395$ and $590 \leq x^{+} \leq 990$ (corresponding to $0 \leq y \leq 1$ and $1.5 \leq x \leq 2.5$ in Fig. 17(c)). 
and wall normal coordinates are defined in wall units. From this picture, we can have a hint on some known important features of boundary layer flows, as (i) the larger aspect ratios and typical inclination of the structures below the onset of the logarithmic layer $\left(y^{+}<30\right)$, (ii) the scaling of structure sizes with their distances to the wall, (iii) the presence of strong vortices which dominate the local velocity fluctuations (there at least, two of these in the picture), and (iv) the fact that the zones of quasi-uniform momentum are correlated with vortex regions, ${ }^{25}$ which in our specific example is particularly clear from the organization of the streamlines in the upper region of the sample $\left(y^{+}>300\right)$.

The streamwise/wall normal plane snapshots of the turbulent channel flow are partitioned in thin streamwise stripes which have vertical width (bin size) $\Delta y^{+} \approx 4$. Through a computational strategy analogous to the one discussed in Sec. IV, we identify vortices for each one of the stripes and determine their mean circulation, peak vorticity, mean radius, and mean number as a function of the stripe distance to the wall. Results are reported in Figs. 19-22. We provide, for some of the pictures, insets which magnify their details, for the sake of better visual inspection.

Similar evaluations of the mean vorticity and mean vortex radii as a function of the distance to the wall have been discussed in Refs. 53 and 54 where, however, vortex parameters are obtained from Levenberg-Marquardt fittings of the identified structures to the Lamb-Oseen vortex pattern. Their results derived from a large turbulent database are compatible with ours, in the context of the $\lambda_{c i}$-criterion.

The application of the $\lambda_{\omega}$-criterion to the turbulent channel DNS data brings a phenomenologically interesting perspective on the statistical properties of the spanwise vortices. It is clear, from Figs. 19-21, that even with the use of the background subtraction procedure, the $\lambda_{c i}$-criterion gives, for all the heights, distinct absolute values of the mean circulations, vorticities, and radii for the populations of positive (retrograde)
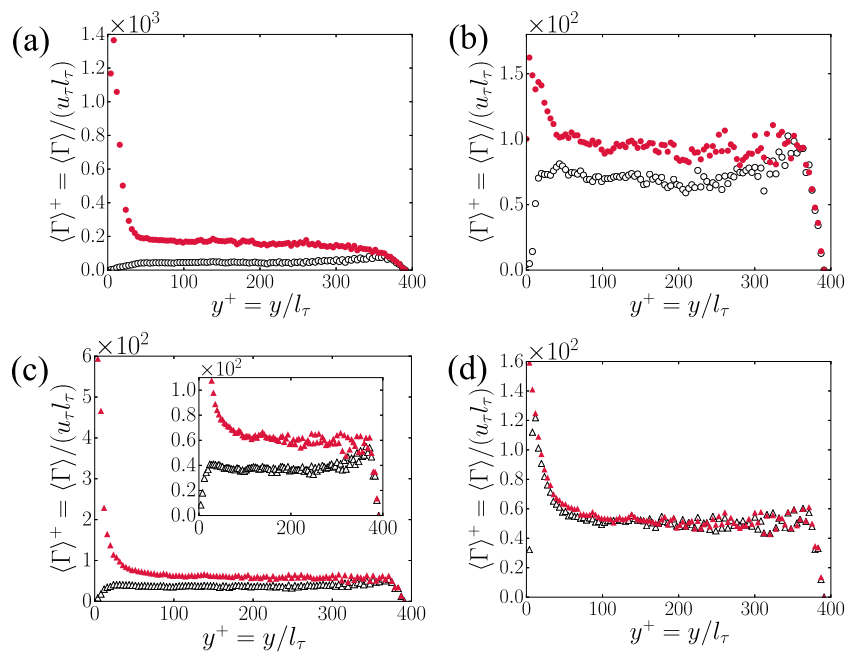

FIG. 19. Absolute mean values of the circulation for retrograde (open symbols) and prograde (solid symbols) vortices, as a function of the distance to the wall. All the quantities are given in wall units (friction velocity $u_{\tau} \simeq 0.34$ and viscous length $l_{\tau} \simeq 2.5 \times 10^{-3}$ ). Plots (a) and (b) are associated to the vortex identification by the $\lambda_{c i}$-criterion, while (c) and (d) are associated to the $\lambda_{\omega}$-criterion. The background subtraction procedure has been applied only for the results depicted in (b) and (d).
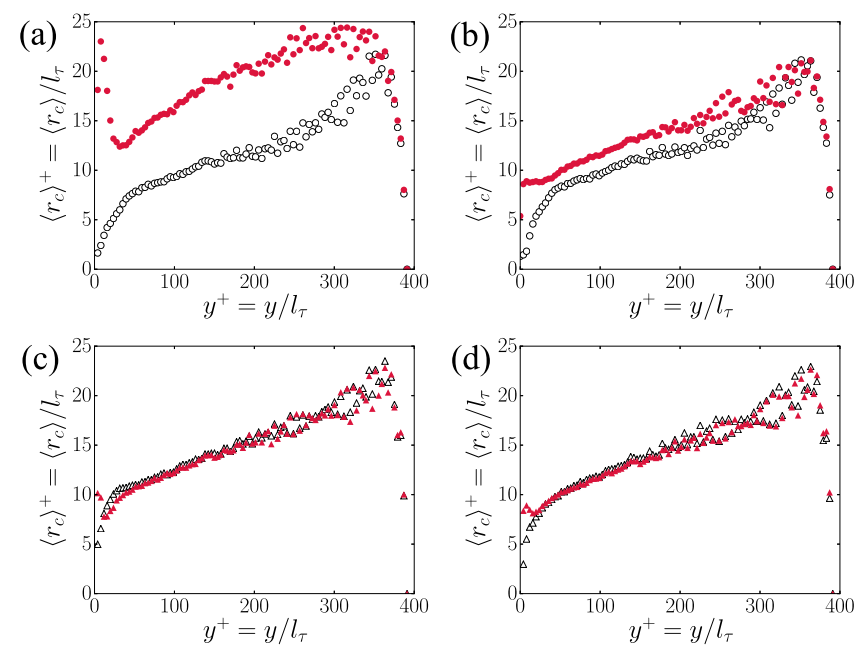

FIG. 20. Mean radius values for retrograde (open symbols) and prograde (solid symbols) vortices, as a function of the distance to the wall. All the rest as in the caption of Fig. 19.

and negative (prograde) vortices. The application of the background subtraction procedure in the $\lambda_{\omega}$-criterion yields, on the other hand, a fine collapse of these quantities for $y^{+}>50$, which extends all throughout the logarithm boundary layer, as it can be appreciated from Figs. 19(d), 20(d), and 21(d). If we now take a look at the populations of prograde and retrograde vortices in Figs. 22(b) and 22(d), they are found to match each other in both criteria, but only after the background subtraction procedure is carried out.

We know, from the law of the wall, that the mean vorticity background is, in the logarithm layer, $\left\langle\omega^{+}\right\rangle=2.5 / y^{+}$. It is clear, thus, from the inspection of Fig. 21, that the mean peak vorticity of the vortex structures is well above the vorticity background value for $y^{+}>50$, which tells us that the there is in fact a weak background shear regime in the log-layer, following the convention put forward in Sec. IV. However, as it is suggested from Fig. 21(d), the buffer layer is likely to be the (a)

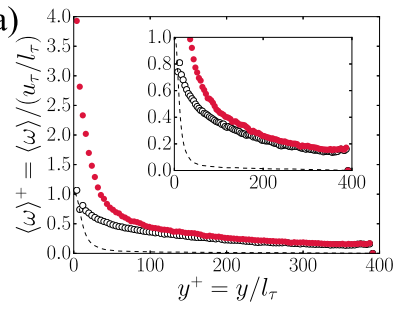

(c)

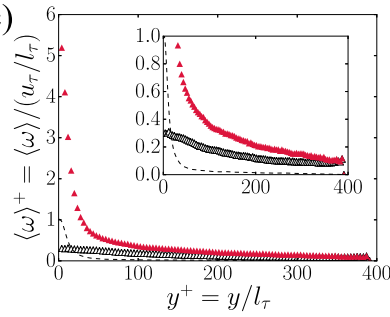

(b)

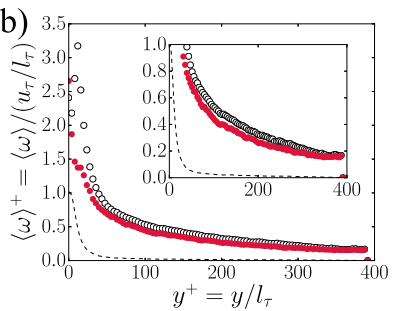

(d)

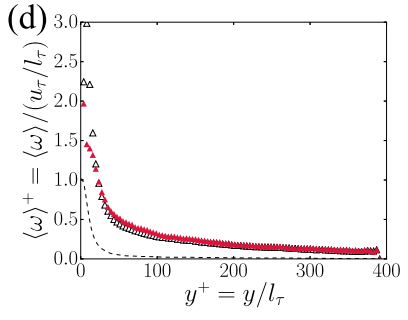

FIG. 21. Absolute mean values of the peak vorticity, i.e., $\left|\left\langle\Gamma / \pi r_{c}^{2}\right\rangle\right|$ for retrograde (open symbols) and prograde (solid symbols) vortices, as a function of the distance to the wall. The dashed line is the average vorticity of the turbulent channel (which closely agrees with the law of the wall). All the rest as in the caption of Fig. 19. 

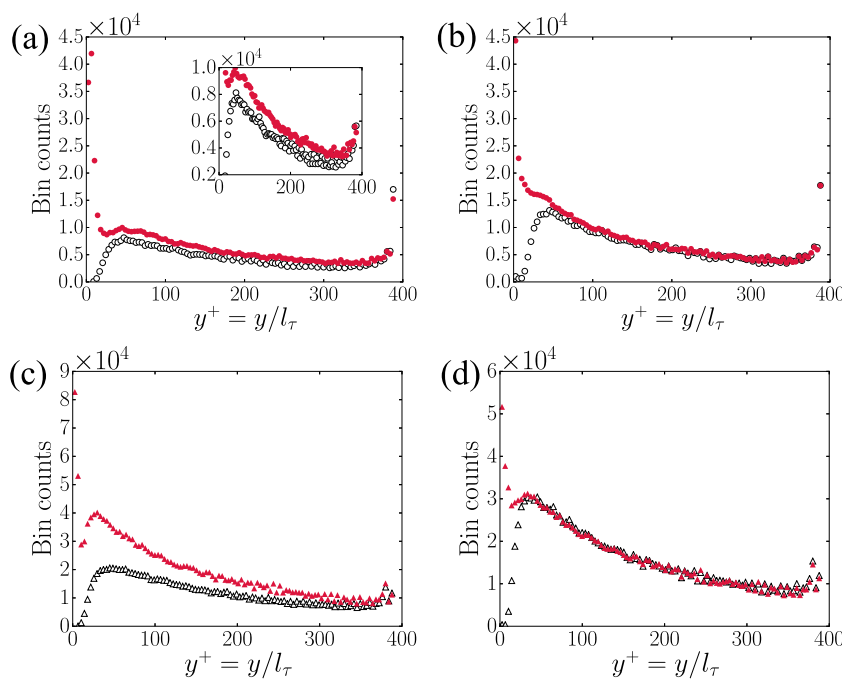

FIG. 22. Vortex counting per stripe of width $\Delta y^{+}=4$, for retrograde (open symbols) and prograde (solid symbols) vortices, as a function of the distance to the wall. All the rest as in the caption of Fig. 19.

region where shear effects can become relevant in the problem of vortex identification.

From the above compilation of statistical results, we find that the detected vortical structures have their vorticities and circulations enhanced within the region $5<y^{+}<30$. This is likely to be related to the observation that near the bottom of the buffer layer, streamwise velocity fluctuations become more intermittent as the distance to the wall decreases, as quantified by a kurtosis analysis. ${ }^{63} \mathrm{~A}$ simple explanation of why individual vortices carry stronger vorticity as they get closer to the wall can be addressed from a combination of the no-slip boundary condition with the attached eddy hypothesis. ${ }^{18}$ It is expected, of course, that fluctuations will disappear deep down in the viscous layer, $y^{+}<5$, which, unfortunately, is poorly resolved in our data.

The data collapse attained in Figs. 19(d), 20(d), 21(d), and 22(d) is an important point for the consolidation of the $\lambda_{\omega}$-criterion, once it supports the long-standing phenomenological assumption of small scale turbulence isotropization in turbulent boundary layers. ${ }^{64-67}$ The $\lambda_{c i}$-criterion yields data collapse only for the vortex counting histogram, Fig. 22(b), failing to do so in the evaluations of vortex circulation, peak vorticity, and radius parameters, as it can be clearly seen from Figs. 19(b), 20(b), and 21(b).

The validity of the isotropic turbulence hypothesis in the turbulent boundary logarithm layer has been usually checked with the help of general theoretical relations that should hold for the expectation values of some local fluid dynamical observables. ${ }^{64-67}$ This is a relevant aspect of the turbulent boundary layer phenomenology that has lacked so far proper corroboration within the structural analyses, a fact due, essentially, to the limitations of the standard $\lambda_{c i}$ vortex identification methodology.

\section{EXTENSION TO THREE-DIMENSIONAL VELOCITY FIELDS}

It is interesting to devise three-dimensional generalizations of the $\lambda_{\omega}$-criterion as a way to investigate the coherent structures that are behind their identified two-dimensional cross sections. There are several ways to do that, following two essential principles that all of the three-dimensional extensions have to satisfy. They have to

(i) be covariant under rotations and

(ii) reduce to the $\lambda_{\omega}$-criterion in two-dimensional slices of the flow.

With the above constraints in mind, let $\vec{\omega}(\vec{r})$ be the threedimensional vorticity vector field, so that we can define, analogously to Eqs. (3.6) and (3.7), the pseudo-velocity and the pseudo-vorticity vector field components, respectively, as

$$
\tilde{v}_{i}(\vec{r})=\epsilon_{i j k} \partial_{j} \omega_{k}(\vec{r})
$$

and

$$
\tilde{\omega}_{i}(\vec{r})=-\partial^{2} \omega_{i}(\vec{r}) .
$$

We can then pick up any of the standard three-dimensional vortex identification methods, like the $Q$ or $\Delta$ criteria, to write down a straightforward generalization of the $\lambda_{\omega}$-criterion. Taking the extensively used $Q$-criterion, ${ }^{27-29}$ as our specific example, recall that

$$
Q\left(\partial_{j} v_{i}\right)=-\frac{1}{2} \partial_{i} v_{j} \partial_{j} v_{i}
$$

Vortex regions are defined as the connected sets of points where $Q>0$. Resorting to the pseudo-velocity and pseudo-vorticity vector fields, the $Q_{\omega}$-criterion, which extends the $\lambda_{\omega}$-criterion to three dimensions, is defined from the scalar field

$$
Q_{\omega}(\vec{r})=\Theta\left(\omega_{i} \tilde{\omega}_{i}\right) Q\left(\partial_{j} \tilde{v}_{i}\right) .
$$

The filtering function previously used in the two-dimensional context is re-written above in terms of the three-dimensional vorticity field. We cannot get rid of it in the definition of the $Q_{\omega}$-criterion, otherwise we would surely recover the vortex identification problems for the cases where the flow is quasi two-dimensional, where $Q_{\omega}(\vec{r})$ becomes essentially equivalent to $\lambda_{\omega}(\vec{r})$, Eq. (3.9).

In the same fashion as it is done with the $Q$-criterion, we look now for the regions of the flow which have $Q_{\omega}>0$ in order to find vortices. The implementation of the background subtraction procedure can be readily done by the substitution of the velocity field by its fluctuation around the mean, exactly as given in the Reynolds decomposition prescription defined by Eq. (4.4).

To contrast the role of locality in the definitions of the $Q$ and the $Q_{\omega}$ criteria, note that we may write, as it is well known, $Q=\left(\Omega_{i j}^{2}-S_{i j}^{2}\right) / 2$, where $\Omega_{i j}$ and $S_{i j}$ are the matrix components of the rotation and the rate of the strain tensors, respectively. Even though the rotation tensor content is identical to the one given by the set of vorticity field components, the $Q$-criterion is, in fact, not fundamentally dependent on the local properties of the vorticity field (as it is the case for the $Q_{\omega}$-criterion). To understand it more clearly, just recall that the strain tensor contribution to $Q$ can be expressed as a non-local functional of the vorticity field, as a direct consequence of Eq. (1.1).

In Fig. 23, we show how the $Q$ and the $Q_{\omega}$ criteria perform for the simulation of the turbulent channel flow considered in Sec. V. As expected, there are many more, and better resolved, 
(a)

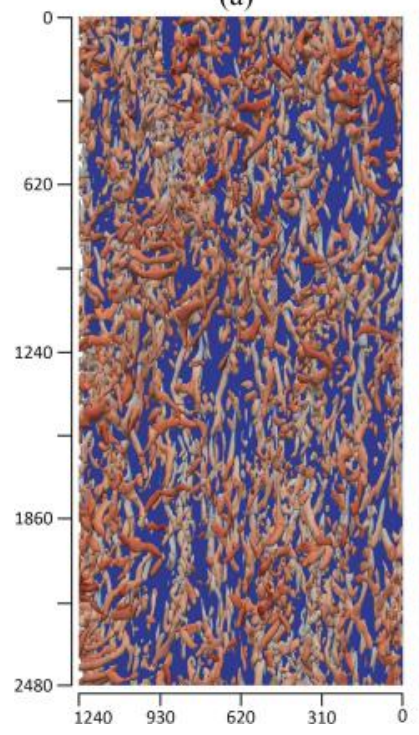

(b)

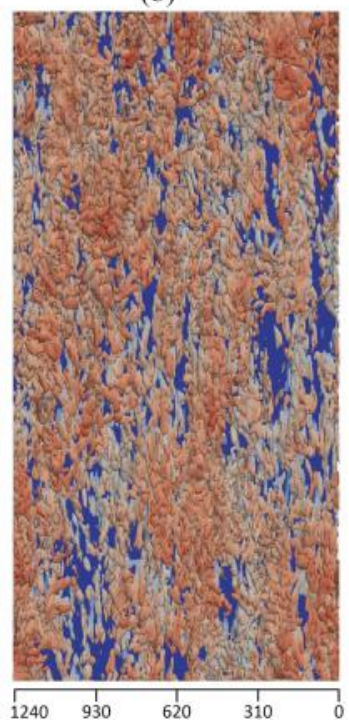

(c)

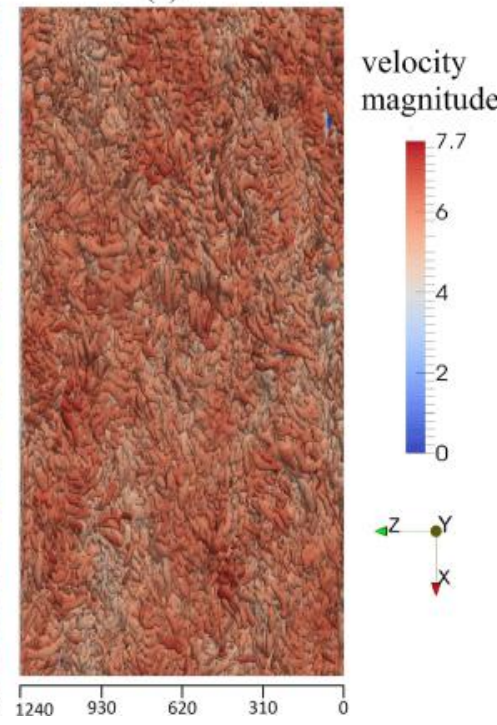

FIG. 23. Vortex identification, with the use of thresholds, as seen from the top of the turbulent channel flow, according to the DNS addressed in Sec. V. (a) $Q$-criterion with no background subtraction, $Q>10^{2}$; (b) $Q_{\omega}$-criterion with background subtraction, $Q_{\omega}>1.1$ $\times 10^{9}$; (c) $Q_{\omega}$-criterion with background subtraction, $Q_{\omega}>1.2 \times 10^{8}$. The color scheme gives the magnitude of the velocity field on the coherent structures. The bottom of the channel is depicted as a uniform blue background. structures obtained from the use of the $Q_{\omega}$-criterion. The color scale indicates the absolute value of the velocity field, which turns out to be a bit more intense for general regions of the flow in the case where the background subtraction procedure has been carried out.

We show, in these pictures, regions which have $Q$ or $Q_{\omega}$ fields greater than the prescribed thresholds, in order to obtain a clear visualization of flow structures at different distances from the wall. Figs. 23(a) and 23(b) are the maps of the coherent structures detected, approximately, for heights $y^{+}<50$, while Fig. 23(c) is related to the structures found within $y^{+}<100$.

The $Q_{\omega}$ images, at variance with the $Q$ ones, suggest long-range correlations between the regions which have higher magnitudes of the velocity field and the presence of vortex packets, a fact that can be related to the existence of the very large-scale motions (VLSMs) observed in the boundary layer flows. ${ }^{68,69}$

Also, when we compare Figs. 23(b) and 23(c), it is tempting to evoke here the conjecture that low speed streaks are connected with the formation of aligned packets of hairpin vortices, as it has been put forward in Ref. 2.

The $Q_{\omega}$-criterion seems, therefore, to be a promising tool to address the three-dimensional organization of vortex structures in the boundary layers at high Reynolds numbers. However, since our aim in this section is just to give a first glimpse on three-dimensional vortex identification, we left this and other interesting issues to further comprehensive studies.

\section{CONCLUSIONS}

We have introduced in this work an alternative vortex identification method-the $\lambda_{\omega}$-criterion (or "vorticity curvature" criterion) - which is fundamentally based on the local properties of the vorticity field. As the starting point of our approach, we have critically revisited the usual swirling strength, $\lambda_{c i}$-criterion, in order to classify its main shortcomings in simple two-dimensional vortex configurations (in two dimensions, most of the velocity gradient-based vortex identification methods become equivalent to the $\lambda_{c i}$-criterion, which, then, has a central status in the general problem of vortex recognition). A careful and rigorous benchmarking analysis has then been carried out, through an extensive statistical Monte Carlo treatment of synthetic vortex systems, in order to compare the performances of the $\lambda_{\omega}$ and the $\lambda_{c i}$ criteria. We have been able to find, in this way, that the $\lambda_{\omega}$-criterion leads, in general, to a considerably better and accurate identification of two-dimensional vortices, as well as of their parameters of circulation, size, and position. We have also shown how to deal with possible spoiling external shear effects, by means of a simple background subtraction procedure, which amounts in the use of the local Reynolds decomposition of the velocity field.

We note that some further, but not very expressive, refinement of the $\lambda_{\omega}$-criterion may be necessary for the cases of moderate/strong background shear in anisotropic vortex distributions (i.e., systems which have more negative than positive vortices, for instance), which may be relevant in flow conditions like the turbulent boundary viscous sublayer.

There are two crucial points that explain the observed good performance of the $\lambda_{\omega}$-criterion: (i) the interesting local properties of the vorticity field, when compared to the ones of the streamfunction (which is a non-local function of vorticity) and (ii) the use of the filtering Heaviside function $\Theta(\omega \tilde{\omega})$ in the definition of the $\lambda_{\omega}$-criterion, as given in Sec. III. This filter removes most of the spurious vortices and renders the vorticity curvature method essentially free from the need of subjective threshold parameters.

We have provided the evidence which supports the application of the $\lambda_{\omega}$-criterion to flow configurations obtained by direct numerical simulations, taking the paradigmatical turbulent channel flow as an example. It turns out that DNS velocity fields are smooth enough to allow the use of the $\lambda_{\omega}$-criterion, a third order derivative scheme. We have been able, in this way, to address the issues of isotropization in 
the turbulent boundary layer, which have, so far, eluded the structural approach. The application of the $\lambda_{\omega}$-criterion to the turbulent channel flow problem has led, for the first time (to the authors' knowledge), to a clear indication of isotropization in the turbulent boundary layer, within the structural point of view. More work is needed here, of course, in combination with the investigation of the three-dimensional coherent structures.

The $\lambda_{\omega}$-criterion is directly generalizable to threedimensions in more than one way. We have explored the three-dimensional extension motivated by the definition of the standard $Q$-criterion, which we have denoted as the " $Q_{\omega}$-criterion." Preliminary visualizations based on the $Q_{\omega}$-criterion show a profusion of well-resolved vortex structures, not revealed in any of the previous standard analyses based on the $Q$-criterion (likely to be affected by both vortex coalescence and erasing due to thresholding), and may shed light on the nature of the VLSMs, once they suggest some correlation between percolating stronger velocity fluctuations and the formation of vortex packets in the turbulent boundary layer.

The study of other important boundary layer aspects is in order, which can now be more accurately addressed. We mean, for instance, an investigation of the coherent structures in the turbulent viscous layer, and their role in the production of viscous drag. In this respect, it is worthwhile mentioning that phenomenological elements like the VLSMs and quasistreamwise vortices, which can be identified with improved resolution through the $Q_{\omega}$-criterion, have been, actually, the subject of previous works focused on the wall shear-stress fluctuations. $^{70,71}$

An interesting discussion, which we touch in passing, leaving a detailed account for a future study, is related to the description of the coherent structures in terms of Kolmogorov scales as developed in Refs. 53 and 61. Consistently with the results of these works, we have found, through an application of the $\lambda_{c i}$-criterion to the streamwise/wall normal planes of our turbulent channel DNS samples, that the Kolmogorovrescaled vortex radii, mean circulations, and mean vorticities become very approximately constant for $y^{+}>50$. This, again, is a strong indication that the local Reynolds number (a function of $y / \eta$ where $\eta$ is the Kolmogorov dissipation length scale) is stable within the large regions of the flow where turbulence can be considered to be effectively isotropic.

To put the bulk of our findings into a proper context, it is important to stress that the $\lambda_{c i}$-criterion (or the $Q$-criterion as well) still offers a reasonably good computational costbenefit ratio for the investigation of high Reynolds number flows, both in experimental and numerical studies. As it can be clearly seen from the turbulent channel analysis put forward in Sec. V, results found from the use of the $\lambda_{c i}$-criterion can be seen as a first approximation to the more accurate ones related to the application of the $\lambda_{\omega}$-criterion, as far as coherent structure resolution and background shear effects are not the points of concern. In such cases, the $\lambda_{c i}$-criterion can be loosely interpreted as a low-pass filtered version of the $\lambda_{\omega}$-criterion.

While the $\lambda_{c i}$-criterion relies on the set of first spatial derivatives of the velocity field and its application to
DNS or Particle Image Velocimetry (PIV) data is, therefore, comparatively less affected by numerical/measurement errors, some special care is necessary when the $\lambda_{\omega^{-}}$ criterion comes into play, once it is a higher-order derivative method.

In order to deal with PIV or DNS data at higher Reynolds numbers, we point out here the main points related to the accuracy of the $\lambda_{\omega}$-criterion. On practical grounds, it is necessary to comply with two basic conditions, namely, (i) the data must be smooth enough to support accurate velocity derivatives up to third order and (ii) the grid resolution has to be fine enough to resolve both the boundaries and interior of the vortex regions. In a general DNS, one can assure that the computations of velocity fields and their second derivatives are accurate if $k^{4} E(k)$, where $E(k)$ is the energy spectrum, is smooth and peaked at inertial range scales. ${ }^{72,73}$ However, the condition (i) can only be achieved if the resolution is high enough so that the tail of the energy spectrum is steeper than $k^{-7}$, which can be sometimes a stringent requirement. Of course, smooth velocity fields can be artificially attained through low-pass filtering, as long as some resolution lost is still acceptable. On the other hand, while condition (ii) is not very problematic in the applications of the swirling strength criterion, which usually produce well resolved large vortex regions, it can be a matter of concern for the vorticity curvature criterion. If the data are already smooth enough, it may be necessary to use a high order interpolation scheme to reach the grid resolution that would resolve vortex domains. In this way, not only PIV but also DNS data may require a careful postprocessing for the use along the lines of the vorticity curvature criterion.

The application of the $\lambda_{\omega}$-criterion to the conventional PIV data can be pursued without much worry when the goal is to study the large scale vortices in the turbulent boundary layer (length scales within and above the logarithmic layer) after the procedure of velocity field smoothing is carried out. Hopefully, smaller structures, within viscous layer dimensions, could be also identified with the help of high resolution PIV data, a subject we deserve for future research.

\section{ACKNOWLEDGMENTS}

We would like to thank D. J. C. Dennis, R. M. Pereira, and J. M. Wallace for the many fruitful discussions during the course of this work. H. Anbarloei is specially acknowledged for his help with the numerical simulations of the turbulent channel flow discussed in this paper. The scientific atmosphere of the Núcleo Interdisciplinar de Dinâmica de Fluidos (NIDF), where part of this work has been developed has been a major source of motivation.

This work has been partially supported by CNPq (Grant No. CT-CNPq/402059/2013-1) and FAPERJ.

${ }^{1}$ S. K. Robinson, "Coherent motions in the turbulent boundary layer," Annu. Rev. Fluid Mech. 23, 601 (1991).

${ }^{2}$ R. J. Adrian, "Hairpin vortex organization in wall turbulence," Phys. Fluids 19, 041301 (2007)

${ }^{3}$ I. Marusic, B. J. McKeon, P. A. Monkewitz, H. M. Nagib, A. J. Smits, and K. R. Sreenivasan, "Wall-bounded turbulent flows at high Reynolds numbers: Recent advances and key issues," Phys. Fluids 22, 065103 (2010). 
${ }^{4}$ J. M. Wallace, "Highlights from 50 years of turbulent boundary layer research," J. Turbul. 13, 53 (2012).

5 J. Jimenez, "Near-wall turbulence," Phys. Fluids 25, 101302 (2013).

${ }^{6} \mathrm{~S}$. Tardu, Coherent Structures in Wall Turbulence (John Wiley \& Sons, 2014).

${ }^{7}$ D. J. C. Dennis, "Coherent structures in wall-bounded turbulence," An. Acad. Bras. Cienc. 87, 2 (2015).

${ }^{8}$ M. Farge, G. Pellegrino, and K. Schneider, "Coherent vortex extraction in 3D turbulent flows using orthogonal wavelets," Phys. Rev. Lett. 87, 054501 (2001).

${ }^{9}$ G. Khujadze, R. N. van Yen, K. Schneider, M. Oberlack, and M. Farge, "Coherent vorticity extraction in turbulent boundary layers using orthogonal wavelets," in Proceedings of the Summer School Program, Center for Turbulence Research, 2010.

${ }^{10}$ K. Yoshimatsu, T. Sakurai, K. Schneider, M. Farge, K. Morishita, and T. Ishihara, "Coherent vorticity in turbulent channel flow: A wavelet viewpoint," in Proceedings of the International Symposium on Turbulence and Shear, 2015.

${ }^{11}$ T. Theodorsen, "Mechanism of turbulence," in Proceedings of the Midwestern Conference on Fluid Mechanics (Ohio State University, Columbus, $\mathrm{OH}$, 1952).

${ }^{12}$ A. A. Townsend, The Structure of Turbulent Shear Flow (Cambridge University Press, 1976).

${ }^{13}$ I. Marusic and R. J. Adrian, "Eddies and scales of wall turbulence," in Ten Chapters in Turbulence, edited by P. A. Davidson, Y. Kaneda, and K. R. Sreenivasan (Cambridge University Press, 2012).

${ }^{14}$ A. E. Perry and M. S. Chong, "On the mechanism of wall turbulence," J. Fluid Mech. 119, 173 (1982).

${ }^{15}$ A. E. Perry, S. M. Henbest, and M. S. Chong, "A theoretical and experimental study of wall turbulence," J. Fluid Mech. 165, 163 (1986).

${ }^{16}$ K. R. Sreenivasan, "A unified view of the origin and morphology of the turbulent boundary layer structure," in Turbulence Management and Relaminarization, edited by H. W. Liepmann and R. Narasimha (Springer-Verlag, 1987).

${ }^{17}$ A. E. Perry and I. Marusic, "A wall-wake model for the turbulence structure of boundary layers. I. Extension of the attached eddy hypothesis," J. Fluid Mech. 298, 361 (1995).

${ }^{18}$ L. Moriconi, "Minimalist turbulent boundary layer model," Phys. Rev. E 79, 046306 (2009).

${ }^{19}$ I. Marusic, R. Mathis, and N. Hutchins, "Predictive model for wall-bounded turbulent flow," Science 329, 193 (2010).

${ }^{20}$ R. Mathis, I. Marusic, S. I. Chernyshenko, and N. Hutchins, "Estimating wall-shear-stress fluctuations given an outer region input," J. Fluid Mech. 715, 163 (2013).

${ }^{21} \mathrm{H}$. Schlicthing and K. Gerstein, Boundary Layer Theory (Springer-Verlag, 2000).

${ }^{22}$ S. B. Pope, Turbulent Flows (Cambridge University Press, 2000).

${ }^{23}$ A. K. M. F. Hussain, "Coherent structure-Reality and myth," Phys. Fluids 26, 2816 (1983).

${ }^{24}$ A. K. M. F. Hussain, “Coherent structures and turbulence," J. Fluid Mech. 173, 303 (1986).

${ }^{25}$ C. D. Meinhart and R. J. Adrian, "On the existence of uniform momentum zones in a turbulent boundary layer," Phys. Fluids 7, 694 (1995).

${ }^{26}$ J. Zhou, R. J. Adrian, and S. Balachandar, "Autogeneration of nearwall vortical structures in channel flow," Phys. Fluids 8, 288 (1996).

${ }^{27}$ A. Okubo, "Horizontal dispersion of floatable particles in the vicinity of velocity singularities such as convergences," Deep-Sea Res. Oceanogr. Abstr. 17, 455 (1970).

${ }^{28}$ J. Weiss, "The dynamics of enstrophy transfer in two-dimensional hydrodynamics," Physica D 48, 273 (1991).

${ }^{29}$ J. C. R. Hunt, A. A. Wray, and P. Moin, "Eddies, stream, and convergence zones in turbulent flows," Center for Turbulence Research Report No. CTRS88, 193 (1988).

${ }^{30}$ M. S. Chong, A. E. Perry, and B. J. Cantwell, "A general classification of three-dimensional flow fields," Phys. Fluids A 2, 765 (1990).

${ }^{31}$ J. Zhou, R. J. Adrian, S. Balachandar, and T. M. Kendall, "Mechanisms for generating coherent packets of hairpin vortices in channel flow," J. Fluid Mech. 387, 353 (1999).

${ }^{32}$ P. Chakabrorty, S. Balachandar, and R. J. Adrian, "On the relationships between local vortex identification schemes," J. Fluid Mech. 535, 189 (2005).

${ }^{33}$ J. Jeong and F. Hussain, "On the identification of a vortex," J. Fluid Mech. 289, 69 (1995).
${ }^{34} \mathrm{P}$. Holmes, J. Lumley, and G. Berkooz, Turbulence, Coherent Structures, Dynamical Systems and Symmetry (Cambridge University Press, 1996).

${ }^{35}$ J. Ferre-Gine, R. Rallo, A. Arenas, and F. Giralt, "Identification of coherent structures in turbulent shear flows with a fuzzy ARTMAP neural network," Int. J. Neural Syst. 7, 559 (1996).

${ }^{36}$ G. Haller, "An objective definition of a vortex," J. Fluid Mech. 525, 1(2005).

${ }^{37}$ G. Haller and F. J. Beron-Vera, "Coherent Lagrangian vortices: The black holes of turbulence," J. Fluid Mech. 731, R4 (2013).

${ }^{38}$ R. J. Adrian, "Conditional eddies in isotropic turbulence," Phys. Fluids 22, 2065 (1979).

${ }^{39}$ R. J. Adrian, "Stochastic estimation of conditional structure: A review," Appl. Sci. Res. 53, 291 (1994).

${ }^{40}$ W. Schoppa and F. Hussain, "New aspects of vortex dynamics relevant to coherent structures in turbulence," in Eddy Structure Identification, CISM Courses and Lectures No. 353, edited by J. P. Bonnet (Springer-Verlag, 1996).

${ }^{41}$ R. J. Adrian, K. T. Christensen, and Z.-C. Liu, "Analysis and interpretation of instantaneous turbulent velocity fields," Exp. Fluids 29, 275 (2000).

${ }^{42}$ P. Chakabrorty, S. Balachandar, and R. J. Adrian, "Kinematics of local vortex identification criteria," J. Visualization 10, 137 (2007).

${ }^{43}$ V. Koláŕ, "Vortex identification: New requirements and limitations," Int. J. Heat Fluid Flow 28, 638 (2007).

${ }^{44}$ Q. Chen, Q. Zhong, M. Qi, and X. Wang, "Comparison of vortex identification criteria for planar velocity fields in wall turbulence," Phys. Fluids 27, 085101 (2015).

${ }^{45}$ G. K. Vallis, Atmospheric and Oceanic Dynamics (Cambridge University Press, 2006)

${ }^{46}$ G. Boffetta, "Two-dimensional turbulence," Annu. Rev. Fluid Mech. 44, 427 (2012).

${ }^{47}$ R. J. Adrian, C. D. Meinhart, and C. D. Tomkis, "Vortex organization in the outer region of the turbulent boundary layer," J. Fluid Mech. 422, 1 (2000)

${ }^{48}$ R. Camussi and F. di Felice, "Statistical properties of vortical structures with spanwise vorticity in zero pressure gradient turbulent boundary layers," Phys. Fluids 18, 035108 (2006).

${ }^{49} \mathrm{Y}$. Wu and K. T. Christensen, "Population trends of spanwise vortices in wall turbulence," J. Fluid Mech. 568, 55 (2006).

${ }^{50}$ D. J. C. Dennis and T. B. Nickels, "Experimental measurement of largescale three-dimensional structures in a turbulent boundary layer. II. Long structures," J. Fluid Mech. 673, 180 (2011).

${ }^{51}$ G. K. Batchelor, Introduction to Fluid Dynamics (Cambridge University Press, 2000).

52 J. A. LeHew, "Spatio-temporal analysis of the turbulent boundary layer and an investigation of the effects of periodic disturbances," Ph.D. thesis, California Institute of Technology, 2012.

${ }^{53}$ S. Herpin, M. Stanislas, and J. Soria, "The organization of near-wall turbulence: A comparison between boundary layer SPIV data and channel flow DNS data," J. Turbul. 11, N47 (2010).

${ }^{54}$ S. Herpin, S. Coudert, J.-M. Foucalt, J. Soria, and M. Stanislas, "Study of vortical structures in turbulent near-wall flows," in Progress in Wall Turbulence: Understanding and Modeling, edited by M. Stanislas, J. Jimenez, and I. Marusic (Springer, 2011).

${ }^{55}$ M. Abramowitz and I. Stegun, Handbook of Mathematical Functions: With Formulas, Graphs, and Mathematical Tables (Dover, 1964).

${ }^{56}$ W. Kühnel, Differential Geometry: Curves - Surfaces - Manifolds (American Mathematical Society, 2006).

${ }^{57} \mathrm{H}$. Mouri, A. Hori, and Y. Kawashima, "Vortex tubes in turbulent velocity fields at Reynolds numbers $\operatorname{Re}_{\lambda} \simeq 300-1300$," Phys. Rev. E 70, 066305 (2004).

${ }^{58}$ N. Hatakeyama and T. Kambe, "Statistical laws of random strained vortices in turbulence," Phys. Rev. Lett. 79, 1257 (1997).

${ }^{59}$ L. Shapiro and G. Stockman, Computer Vision (Prentice Hall, 2001).

${ }^{60}$ Eddy exclusion seems to be a realistic feature of boundary layer flows. See C. M. de Silva, J. D. Woodcock, N. Hutchins, and I. Marusic, "Influence of spatial exclusion on the statistical behaviour of attached eddies," Phys. Rev. Fluids 1, 022401(R) (2016).

${ }^{61}$ M. Tanashi, S.-J. Kang, T. Miyamoto, S. Shiokawa, and T. Miyauchi, "Scaling law of fine scale eddies in turbulent channel flows up to $R e_{\tau}=800$," Int. J. Heat Fluid Flow 25, 331 (2004).

${ }^{62}$ J. Kim, P. Moin, and R. Moser, "Turbulence statistics in fully developed channel flow at low Reynolds number," J. Fluid Mech. 177, 133 (1987). 
${ }^{63}$ T. Lorkowski, "Small-scale forcing of a turbulent boundary layer," FDRL TR 97-2, Fluid Dynamics Research Laboratory, Massachusetts Institute of Technology, 1997.

${ }^{64} \mathrm{~S}$. Corrsin, Local isotropy in turbulence shear flow, Research Memo 58B11, NACA, 1958

${ }^{65}$ S. G. Saddoughi and S. V. Veeravalli, "Local isotropy in turbulent boundary layers at high Reynolds number,” J. Fluid Mech. 268, 333 (1994).

${ }^{66} \mathrm{G}$. Xu, S. Rajagopalan, and R. A. Antonia, "Approach to isotropy in a smooth wall turbulent boundary layer," Fluid Dyn. Res. 26, 1 (2000).

${ }^{67}$ G. Gulitski, M. Kholmyansky, W. Kinzelbach, B. Luthi, A. Tsinober, and S. Yorish, "Velocity and temperature derivatives in high-Reynolds-number turbulent flows in the atmospheric surface layer. I. Facilities, methods and some general results," J. Fluid Mech. 589, 57 (2007).

${ }^{68}$ K. C. Kim and R. J. Adrian, "Very large-scale motion in the outer layer," Phys. Fluids 11, 417 (1999).
${ }^{69}$ S. C. C. Bailey and A. J. Smits, "Experimental investigation of the structure of large-and very-large-scale motions in turbulent pipe flow," J. Fluid Mech. 651, 339 (2010).

${ }^{70}$ A. G. Kravchenko, H. Choi, and P. Moin, "On the relation of near-wall streamwise vortices to wall skin friction in turbulent boundary layers," Phys. Fluids A 5, 3307 (1993).

${ }^{71}$ H. Abe, H. Kawamura, and H. Choi, "Very large-scale structures and their effects on the wall shear-stress fluctuations in a turbulent channel flow up to $\operatorname{Re}_{\tau}=640$," J. Fluid Eng. 126, 835(2004).

${ }^{72}$ H. Abe, R. A. Antonia, and H. Kawamura, "Correlation between small-scale velocity and scalar fluctuations in a turbulent channel flow," J. Fluid Mech. 627, 1 (2009).

${ }^{73}$ A. W. Vreman and J. G. M. Kuerten, "Statistics of spatial derivatives of velocity and pressure in turbulent channel flow," Phys. Fluids 26, 085103 (2014). 